\title{
Periphytic Diatoms in the Polluted Linggi (sensu stricto) and Kundor Rivers, Negeri Sembilan, Malaysia
}

\author{
I.S.A. NATHER KHAN
}

\begin{abstract}
The investigation in this paper aimed to describe periphytic diatom assemblage, species composition and distribution in tropical polluted Linggi (sensu stricto or s.s.) and Kundor rivers in Negeri Sembilan, Malaysia. Regardless of ecological and environmental conditions, diatoms were the numerically dominant flora among periphytic algae collected monthly over a period of 13 months at nine stations in the Linggi (s.s.) and Kundor rivers. The freshwater periphytic diatom samples were collected mainly using artificial substrates and supplemented with natural substrates. The periphytic diatoms thus collected from both natural and artificial substrates comprised 86 taxa ( 82 pennate and 4 centric forms) belonging to 21 genera. Of the 86 species, 71 species were found colonized in artificial substrates while the remaining 15 species were recorded exclusively on natural substrates. On the whole, the most common diatoms in both rivers combined were Eunotia vanheurckii, Gomphonema parvulum, Nitzschia palea, Pinnularia braunii, Navicula cryptocephala, Achnanthes saxonica, Achnanthes minutissima and Pinnularia microstauron. The most abundant species were E. vanheurckii, N. palea, A. saxonica, G. parvulum and A. minutissima.
\end{abstract}

Key words: Diatom, periphyton, biological assessment, aquatic ecology, lotic ecosystem, tropical river, river ecology, water pollution, water quality, Malaysia

There is a general paucity of scientific research on taxonomy, species composition and distribution of freshwater periphytic diatoms in Malaysia. Prowse (1962) provided the sole detailed taxonomic work on diatoms, although there were many general limnological studies that were carried out in Malaysian rivers, lakes and ponds covered some aspects of diatoms (Hirano 1967; Mizuno \& Mori 1970; Prowse \& Ratnasabapathy 1970; Arumugam 1972; Furtado \& Mori 1982; Nather Khan \& Haji Mohamed 1985). Bishop (1973) and Ho (1973) included freshwater algae in their studies on river ecology and attempted to correlate diatoms distribution with water quality and other ecological factors. Nather Khan (1985) conducted an extensive study on biological assessment of water pollution using diatoms community structure, species diversity and productivity in the Linggi River Basin (Nather Khan 1990a, b, c; 1991a, b; 1992a, b; Nather Khan et al. 1986a, b; 1987); Nather Khan \& Lim 1991; Nather Khan \& Firuza 2010, 2012).

Algae which live attached to surfaces of river substrates are called periphyton or Aufwuchs. Periphytons are important primary producers in lotic compared to lentic ecosystems where phytoplanktons are major primary producers. Normally diatoms are numerically most dominant algal group among periphyton community in lotic system and have been found to be reliable indicators of water pollution. In order to overcome difficulty in collecting

Ecotone Environmental Management Sdn. Bhd., Suite 912, Block A, Kelana Centre Point, Kelana Jaya, 47301 Petaling Jaya, Selangor, D.E., Malaysia

* Corresponding author (e-mail: ecotonemalaysia@yahoo.com) 
quantitative samples from natural substrates, artificial substrates such as pieces of wood, sterilized smooth stones, plastic sheets or glass slides were used for quantitative assessment of periphytic diatoms in lotic environment (Sladecek \& Sladekova 1964; King \& Ball 1967; Bishop 1973; Nather Khan 1985; Nather Khan et al. 1987).

As diatoms were numerically abundant in periphyton community in the Linggi (s.s.) and Kundor rivers qualitative and quantitative assessment of diatom community was estimated through microscopic counting methods (Nather Khan et al. 1987). The investigation aimed to determine and describe the taxonomy, species composition, distribution, diversity, relative abundance and productivity of periphytic diatom community with reference to prevailing pollution and water quality in the rivers (Nather Khan 1990a; Nather Khan 1990b; 1991a; 1991b; 1992a; 1992b; Nather Khan et al. 1986a; 1986b; 1987; 1991; 2010; 2012). However, this paper describes mainly the taxonomy and distribution of periphytic diatoms at variatous locations at the Linggi (s.s.) and Kundor rivers with reference to water pollution.

\section{MATERIALS AND METHODS}

\section{The Linggi (s.s.) and Kundor Rivers}

The Linggi (s.s.) and Kundor were highly polluted sub-basins of Linggi River Basin, and located at $2^{\circ} 24^{\prime}-2^{\circ} 50^{\prime} \mathrm{N}$ latitude and $101^{\circ} 53^{\prime}-102^{\circ} 12^{\prime} \mathrm{E}$ longitude at south-western part of the state of Negeri Sembilan. The Linggi sub-basin has more than 21 major tributaries, of which seven are located above Seremban town, the state capital. The predominant types of land use in the basin were rubber and oil palm plantations, small areas of rice fields with urban and industrial areas. Water from these rivers were extensively used for domestic, industrial and irrigation purposes. The river sections under investigation at the Linggi (s.s.) river received mostly treated and untreated urban and industrial wastes from Seremban municipality area, though the domestic sanitary wastes from Seremban town ranked highest among all pollutants discharged.

The Kundor, a tributary of Pedas subbasin was highly polluted tributary within the Linggi River Basin which was due to effluent discharged from rubber and oil palm factories from Ulu Kanchong estate. The upstream section of this tributary runs through rubber and oil palm plantations while the downstream section passes through lowland swampy areas including rice paddy fields, where a large volume of water was extracted from several small reservoirs built over the Kundor river for paddy irrigation.

\section{Water Quality and Periphtyic Diatom Sampling Stations}

Twelve sampling stations were established at both the rivers to assess water quality on monthly basis over 13 months. Of the twelve sampling stations, eight stations were selected only for quantitative periphytic diatom study. The first four stations (Stations $1-4$ ) were located at the Linggi (s.s.) river while remaining four stations (Stations 9 - 12) were located at the Kundor river. The locations and general characteristic features of these stations are depicted in Figure 1. When selecting sampling stations, steps were taken to minimise habitat heterogeneity and other ecological variation between the stations. The dates, sampling location, sampling methods, effluent type and load discharged were described in detail in several other papers published earlier (Nather Khan 1990a; 1990b; 1990c; 1991a; 1991b; 1992a; 1992b).

\section{Water Quality Measurement}

Apart from monitoring river flow and river discharge, water samples were collected on a monthly basis at all the stations for a period of 13 months, from January 1983 to January 


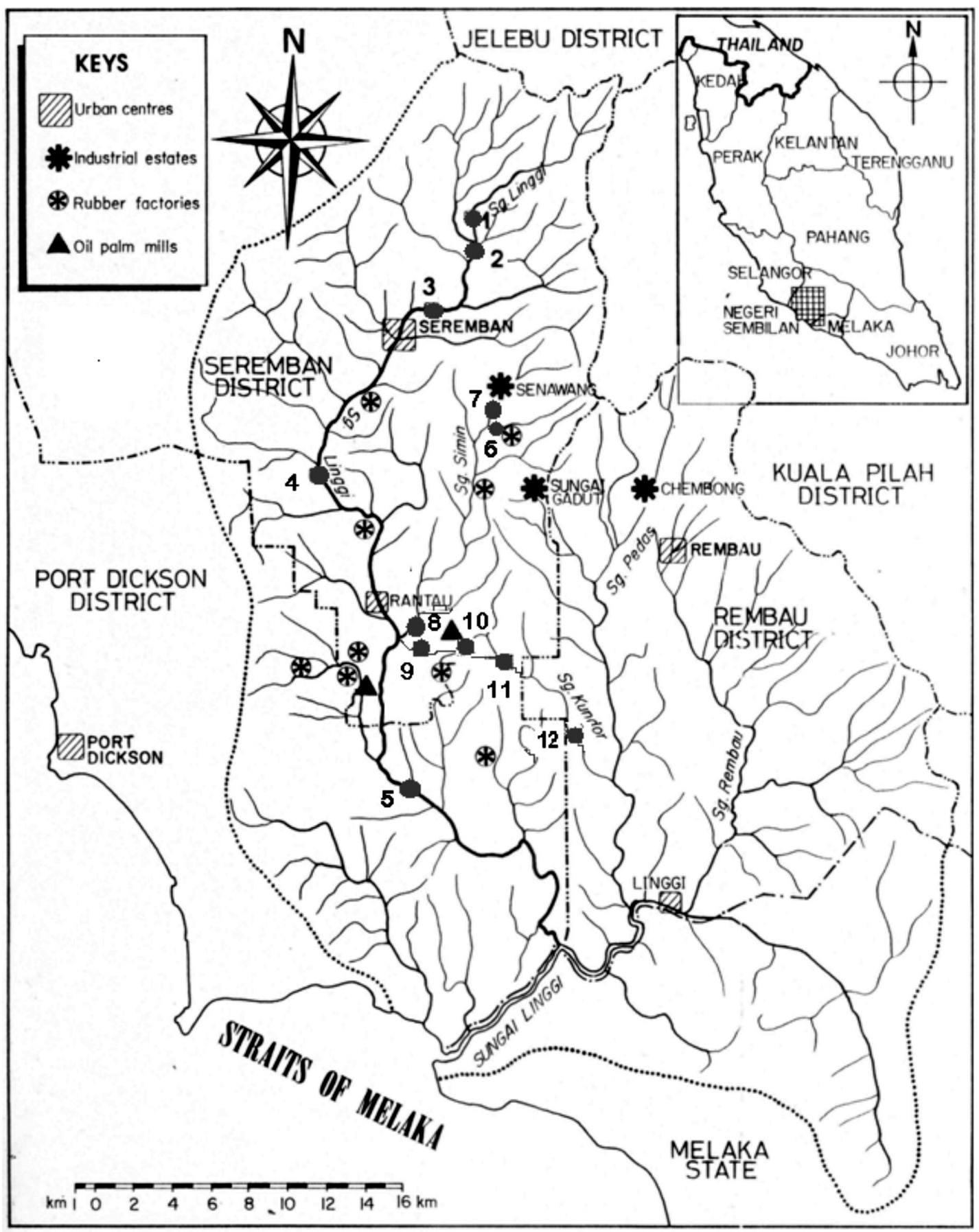

Figure 1. Sampling stations at Linggi (s.s.) and Kundor rivers of Linggi River Basin. 
1984 to analyse 29 physical and chemical parameters. Water samples were collected from middle of the water column using prewashed polyethylene bottles. The temperature, $\mathrm{pH}$, and conductivity were measured in the field and dissolved oxygen was determined immediately upon returning to laboratory. All samples were analysed within $48 \mathrm{hrs}$ after collection except the biochemical oxygen demand (BOD). All determinations were made in duplicate and repeated when precision was needed. The physical and chemical parameters analysed were based on methods outlined in APHA (1975) and Mackereth et al. (1978). For details of the methods employed and extensive data collected were discussed in other earlier publications related to the studies (Nather Khan 1992a \& 1992b).

\section{Diatoms Sampling Methods}

Diatoms were sampled from both natural and artificial substrates. Old leaves, stems and roots of submerged marginal plants were randomly collected within 10 metre river reaches of each station and preserved immediately in 80 $\mathrm{ml}$ of $4 \%$ formalin in wide mouthed bottles. Additional samples were collected from stones and rocks. Samples thus collected from natural substrates were used for qualitative study only, particularly for species composition and identification purposes (Whitford \& Schumacher 1963; Nather Khan 1990a).

For detailed quantitative assessment, diatom samples were collected using glass microscope slides as artificial substrates from January 1983 until January 1984 at Stations 1 to 4 and from March 1983 until January 1984 at Stations 9 to 12. A special diatom collection device was constructed using wooden frames to hold 12 microscope slides $(75 \times 25 \mathrm{~mm})$. At each station two such devices, one at each side of bank of the river were placed and held vertically parallel to the current and just below the water level by means of iron stakes and wire. Approximately after a month, exposed frames were removed and replaced with new frames for the next colonisation period. Two colonized slides were randomly chosen from frames and preserved immediately in $80 \mathrm{ml}$ of $4 \%$ formalin. Additional slides were taken to laboratory in bottles containing river water to examine diatoms in fresh form.

\section{Diatom Identification and Enumeration}

In the laboratory, two slides preserved in formalin were scrapped to remove diatom colony and were 'cleaned' by adding nitric acid and potassium dichromate as described by Hohn and Hellerman (1963). The cleaned samples were made up to known quantities and one $\mathrm{ml}$ of aliquot of acid-free, homogenised suspension was placed on a $22 \mathrm{ml} \# 1$ cover glass, dried on a hot plate and mounted on a slide with Hyrax mounting medium. In order to determine relative abundance and diversity of diatom species a numerical counting method was used. Each slide was randomly scanned until 500 diatom cells were counted under a magnification of $\times 600$. For identification, diatom frustules were examined under oil immersion $(\times 1000$ magnification). The count data for each species were expressed as number of individuals per 500 cells counted. As far as possible, diatoms were identified to species level using taxonomic keys, drawings and descriptions given in such works as Prowse (1962), Sladecek (1963), Mizuno (1964), Patrick \& Reimer (1966) and Ho (1973).

\section{RESULTS AND DISCUSSION}

\section{Water Quality at Linggi (s.s.) and Kundor Rivers}

The water quality measurement carried out over a year indicated that Station 1 and 2 were characterised by relatively low ionic content, slightly neutral $\mathrm{pH}$, high silica and oxygen contents, low BOD and permanganate values (Table 1). The ammonia-nitrogen, nitrates 


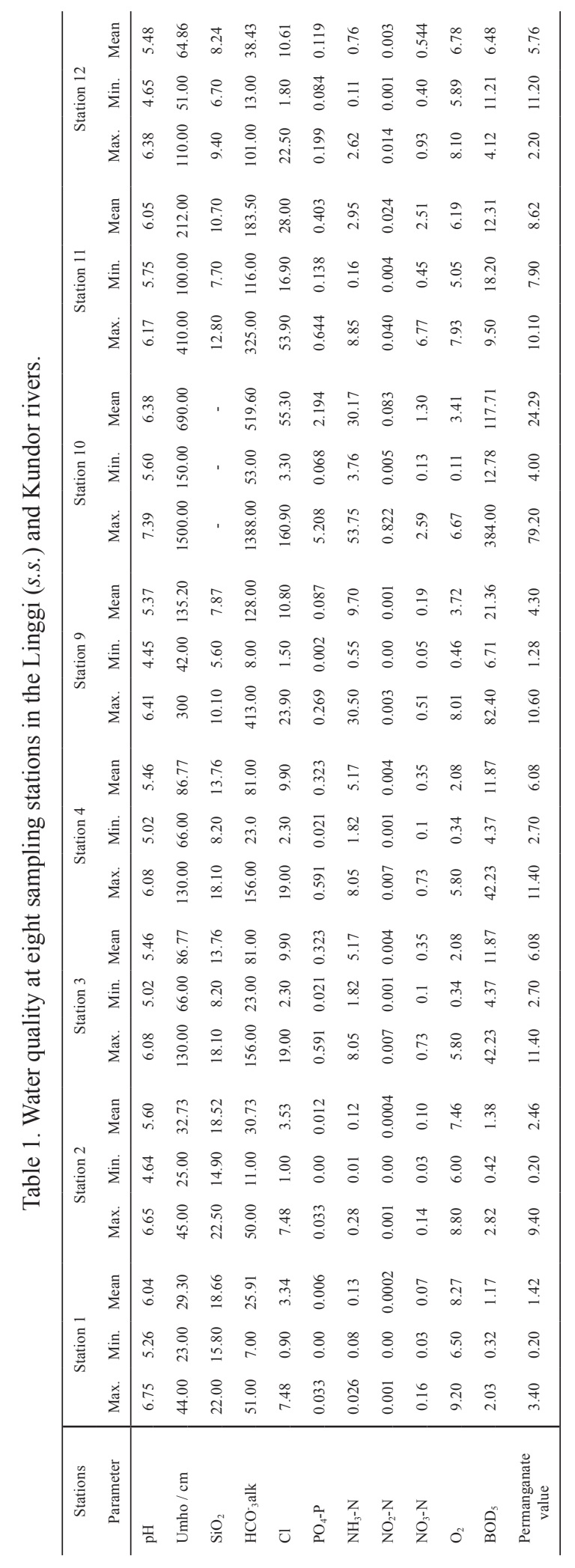


and phosphates were very low and nitrites were usually present in low or sometimes in undetectable level. Drastic changes in the above physical and chemical parameters were found at Station 3. Very high specific conductivity, ammonia, nitrate and BOD were recorded at this station. This was due to effluent discharged from nearby rubber processing factory. The water quality at Station 4, located south of the Seremban town, revealed increased concentration of substances associated with sewage and its decomposition products. The concentration of dissolved oxygen was reduced to a very low level here. This was due to the large amount of treated and untreated sewage discharged from Seremban town upstream of the Station 4.

Among all the stations, very high physical and chemical values were recorded at Stations 9, 10, 11 and 12 that located at Kundor river (Table 1). The conductivity, alkalinity, chloride, ammonical nitrogen, BOD and permanganate values were high at almost all the stations. This was due to oil palm effluent discharged into the river of low dilution capacity. Based on the concentrations of some important water quality parameters, Station 1 and 2 could be considered unpolluted, Station 3 moderately polluted and Stations 4, 9,10, 11 and 12 were severely polluted, though there was a great variation in pollution type and load, water quality river flow and discharge between these stations.

\section{Species Composition of Periphytic Diatoms}

The diatoms collected from both natural and artificial substrates comprised 86 taxa ( 82 pennate and 4 centric forms) belonging to 21 genera (Table 2). Of the 86 species, 71 species were found colonized in artificial substrates while the remaining 15 species were recorded exclusively on natural substrates. However, these 15 species were very rare and appeared only at certain stations (Stations 1, 2, 3 and 7 ) in certain months of the year (Nather Khan 1990b). The number of diatom species observed between stations varied from 22 to 47 species under $8-16$ genera respectively. Stations 1, 2 and 3 had more than $50 \%$ of all species recorded. The maximum numbers of species recorded at Station 1 were 47 and the minimum number of species was recorded at Station 6 were 22. Out of 71 species observed on artificial substrates, 43 species were recorded at Station 1, 42 species at Stations 2 and 3; 27 species at Station 4; 22 species at Station 9; 26 Species at Station 10 and 34 species at Stations 11 and 12 together (Tables 3 and 4). On the whole, the annual mean number of species varied from 8 to 22; the minimum ( 8 species) at Station 9 and the maximum (22 species) at Station 3.

Almost all species of genera Achnanthes, Synedra and several species of genera Cymbella, Gomphonema and Surirella were recorded at Station 1. Several species of Eunotia were recorded at Station 2, several species of Navicula at Station 3, several species of Pinnularia at Station 6 and several species of Nitzschia at Station 7. Among all the genera, Navicula was the most dominant genus with 13 species followed by Cymbella and Eunotia with 9 species each while Achnanthes and Nitzschia were the next with 8 species each.

\section{Spatial Variation of Periphytic Diatoms}

The occurrence and abundance of diatoms varied from station to station. Within a few days of exposure at Station 1 thin layer of mucilage with scattered individuals of Achnanthes minutissima, Cymbella javanica and Synedra rumpens were found, while at Stations 2 and 3, A. minutissima and Achnanthes saxonica were the initial colonizers. A. saxonica and A. minutissima were two most common and abundant species at Station 1 comprising over $75 \%$ of cell counts. Both these species showed $100 \%$ constancy values which meant, these species were present in all the sampling months. The other common species at Station 
Table 2. Periphytic diatoms flora in the Linggi (s.s.) and Kundor rivers.

\begin{tabular}{|c|c|}
\hline No & Name of the species \\
\hline 1 & Achnanthes minutissima Kuetz. var. minutissima \\
\hline 2 & Achnanthes linearis (W. Sm.) Grun. var. linearis \\
\hline 3 & Achnanthes saxonica Krasske \\
\hline 4 & Achnanthes lapidosa var. lanceolata Hustest \\
\hline 5 & Achnanthes stauroneiformis Prowse \\
\hline 6 & Achnanthes crenulata Grun. * \\
\hline 7 & Achnanthes brevipes C.A. Agardh var. intermedia Kuetz. \\
\hline 8 & Achnanthes exigua Grun. \\
\hline 9 & Amphora normani Rabenhorst \\
\hline 10 & Cyclotella meneghiniana Kuetz. \\
\hline 11 & Cyclotella glomerata Bachmann \\
\hline 12 & Cocconeis thumensis A. Mayer \\
\hline 13 & Cymbella javanica Hustedt \\
\hline 14 & Cymbella sumatrensis Hustedt \\
\hline 15 & Cymbella turgida Gregory \\
\hline 16 & Cymbella japonica Reichert* \\
\hline 17 & Cymbella minuta Hilse. ex. Rabh. var. minuta * (Syn. C. ventricosa) \\
\hline 18 & Cymbella sumatrensis fo. malayensis nov. fo (Syn. C. lanceolata) \\
\hline 19 & Cymbella kolbei Hustedt \\
\hline 20 & Cymbella tumida (Brebisson) van. Heurek. * \\
\hline 21 & Cymbella cuspidata Kuetz. \\
\hline 22 & Diploneis ovalis (Hilsse) P. T. Cleve \\
\hline 23 & Desmogonium rabenhorstianum Grun. * \\
\hline 24 & Eunotia monodon Ehrenberg var. constricta A. Cleve-Euler \\
\hline 25 & Eunotia vanheurckii Patr. var. vanheurckii* (Syn.E.faba (Ehr.) Grun. \\
\hline 26 & Eunotia rhomboidea Hustest * \\
\hline 27 & Eunotia major (W. Smith) Rabenhorst var. indica (Grun.) A. Berg \\
\hline 28 & Eunotia pectinalis (Kuetz) Rabenhorst* \\
\hline 29 & Eunotia polydentula (Brun) A. Berg. var. perminuta Grun. lunaris (Ehr.) Grun. var. capitata Grun. \\
\hline 30 & Eunotia lunaris (Ehr.) Grun. var. capitata Grun \\
\hline 31 & Eunotia camelus Ehr. var. karveerensis Gandhi \\
\hline 32 & Eunotia major (W. Smith) Rabenhorst \\
\hline 33 & Fragilaria vauncheriae (Kuetz) Boye Petersen \\
\hline 34 & Fragilaria sp. * \\
\hline 35 & Frustulia rhomboides (Ehr.) de Toni \\
\hline 36 & Frustulia saxonica Rabenhorst \\
\hline 37 & Frustulia javanica Hustedt * \\
\hline 38 & Gomphonema parvulum (Kuetz) Grun. \\
\hline 39 & Gomphonema gracile Ehr. \\
\hline 40 & Gomphonema subventricosum Hustedt \\
\hline 41 & Gomphonema longiceps Ehr. var. subclavata Grun. F. gracilis Hustedt \\
\hline 42 & Gomphonema clevei var. inaequilongum $\mathrm{H}$. Kobayasi \\
\hline 43 & Gyrosigma attenuatum (Kuetz) P.T. Cleve \\
\hline 44 & Melosira granulata (Ehr.) Ralfs \\
\hline 45 & Melosira italica (Ehr.) Kuetz. \\
\hline
\end{tabular}


Table 2 (Cont.). Periphytic diatoms flora in the Linggi (s.s.) and Kundor rivers.

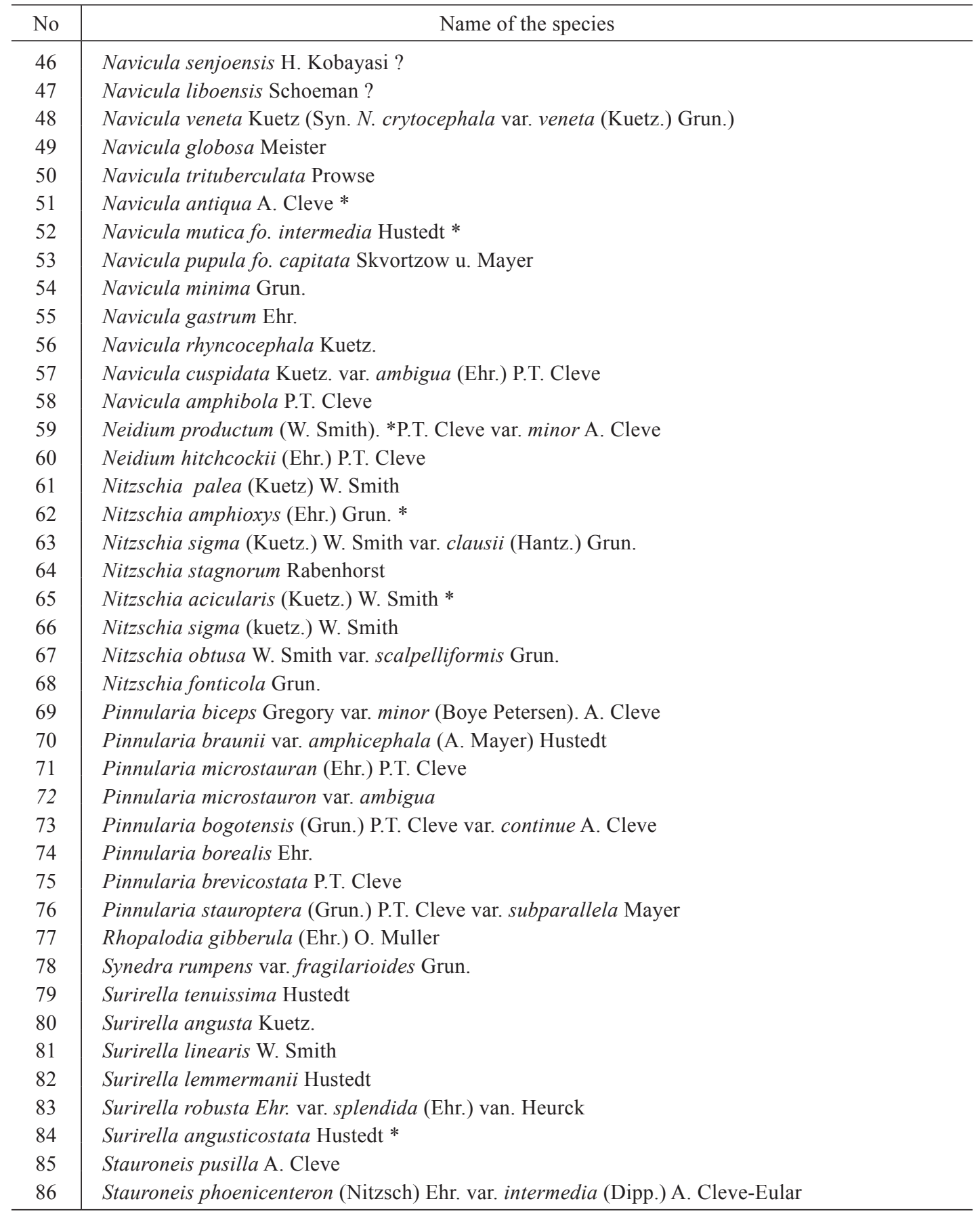

$\left(^{*}\right)$ indicates species recorded exclusively from natural substrates. 
Table 3. Periphytic diatom occurrence at various stations (in alphabetical order).

\begin{tabular}{|c|c|c|c|c|c|c|c|c|}
\hline \multirow{3}{*}{ No } & \multirow{3}{*}{ Diatom species } & \multicolumn{7}{|c|}{ Stations } \\
\hline & & \multicolumn{4}{|c|}{ Linggi (s.s) River } & \multicolumn{3}{|c|}{ Kundur River } \\
\hline & & St. 1 & St. 2 & St. 3 & St. 4 & St. 9 & St. 10 & St. $11 \& 12$ \\
\hline 1 & Achnanthes minutissima & $\sqrt{ }$ & $\sqrt{ }$ & $\sqrt{ }$ & $\sqrt{ }$ & $\sqrt{ }$ & $\sqrt{ }$ & $\sqrt{ }$ \\
\hline 2 & Achnanthes linearis & $\sqrt{ }$ & $\sqrt{ }$ & $\sqrt{ }$ & $\sqrt{ }$ & $\sqrt{ }$ & $\sqrt{ }$ & - \\
\hline 3 & Achnanthes saxonica & $\sqrt{ }$ & $\sqrt{ }$ & $\sqrt{ }$ & $\sqrt{ }$ & $\sqrt{ }$ & $\sqrt{ }$ & - \\
\hline 4 & Achnanthes lapidosa & $\sqrt{ }$ & $\sqrt{ }$ & - & - & - & - & - \\
\hline 5 & Achnanthes stauroneiformis & $\sqrt{ }$ & - & $\sqrt{ }$ & - & - & - & - \\
\hline 6 & Achnanthes crenulata* & $\sqrt{ }$ & - & - & - & - & - & - \\
\hline 7 & Achnanthes brevipes & $\sqrt{ }$ & $\sqrt{ }$ & $\sqrt{ }$ & - & - & - & - \\
\hline 8 & Achnanthes exigua & $\sqrt{ }$ & $\sqrt{ }$ & - & $\sqrt{ }$ & - & $\sqrt{ }$ & $\sqrt{ }$ \\
\hline 9 & Amphora normani & $\sqrt{ }$ & - & $\sqrt{ }$ & $\sqrt{ }$ & - & - & - \\
\hline 10 & Cyclotella meneghiniana & - & $\sqrt{ }$ & $\sqrt{ }$ & $\sqrt{ }$ & - & - & $\sqrt{ }$ \\
\hline 11 & Cyclotella glomerata & $\sqrt{ }$ & $\sqrt{ }$ & - & - & - & - & - \\
\hline 12 & Cocconeis thumensis & - & $\sqrt{ }$ & - & - & - & - & - \\
\hline 13 & Cymbella javanica & $\sqrt{ }$ & $\sqrt{ }$ & $\sqrt{ }$ & $\sqrt{ }$ & $\sqrt{ }$ & - & $\sqrt{ }$ \\
\hline 14 & Cymbella sumatrensis & $\sqrt{ }$ & $\sqrt{ }$ & $\sqrt{ }$ & - & - & - & - \\
\hline 15 & Cymbella turgida & $\sqrt{ }$ & $\sqrt{ }$ & $\sqrt{ }$ & - & - & - & $\sqrt{ }$ \\
\hline 16 & Cymbella japonica* & $\sqrt{ }$ & - & - & - & - & - & - \\
\hline 17 & Cymbella minuta* & $\sqrt{ }$ & $\sqrt{ }$ & - & - & - & - & - \\
\hline 18 & Cymbella sumatrensis fo. malayensis & $\sqrt{ }$ & - & - & $\sqrt{ }$ & - & - & - \\
\hline 19 & Cymbella kolbei var. lanceolata & $\sqrt{ }$ & - & $\sqrt{ }$ & - & - & $\sqrt{ }$ & - \\
\hline 20 & Cymbella tumida* & $\sqrt{ }$ & - & - & - & - & - & - \\
\hline 21 & Cymbella cuspidata & - & - & - & $\sqrt{ }$ & - & - & - \\
\hline 22 & Diploneis ovalis & $\sqrt{ }$ & - & $\sqrt{ }$ & - & - & - & - \\
\hline 23 & Desmogonium rabenhorstianum* & - & $\sqrt{ }$ & - & - & - & - & - \\
\hline 24 & Eunotia monodon & $\sqrt{ }$ & $\sqrt{ }$ & $\sqrt{ }$ & $\sqrt{ }$ & $\sqrt{ }$ & $\sqrt{ }$ & $\sqrt{ }$ \\
\hline 25 & Eunotia vanheurckii* & - & - & - & - & - & - & $\sqrt{ }$ \\
\hline 26 & Eunotia rhomboidea* & - & $\sqrt{ }$ & - & - & - & - & - \\
\hline 27 & Eunotia major & - & $\sqrt{ }$ & $\sqrt{ }$ & $\sqrt{ }$ & $\sqrt{ }$ & - & - \\
\hline 28 & Eunotia pectinalis* & - & $\sqrt{ }$ & - & - & - & - & - \\
\hline 29 & Eunotia polydentula & - & - & $\sqrt{ }$ & - & - & - & - \\
\hline 30 & Eunotia lunaris & - & - & - & - & $\sqrt{ }$ & - & - \\
\hline 31 & Eunotia camelus & - & - & - & - & - & $\sqrt{ }$ & - \\
\hline 32 & Eunotia major var. indica & - & - & - & - & - & - & $\sqrt{ }$ \\
\hline 33 & Fragilaria vaucheriae & $\sqrt{ }$ & - & - & - & - & - & - \\
\hline 34 & Fragilaria sp. ${ }^{*}$ & $\sqrt{ }$ & - & - & - & - & - & - \\
\hline 35 & Frustulia rhomboides & - & - & $\sqrt{ }$ & - & - & - & - \\
\hline 36 & Frustulia saxonica & $\sqrt{ }$ & $\sqrt{ }$ & $\sqrt{ }$ & $\sqrt{ }$ & $\sqrt{ }$ & $\sqrt{ }$ & $\sqrt{ }$ \\
\hline 37 & Frustulia javanica* & - & $\sqrt{ }$ & - & - & - & - & - \\
\hline 38 & Gomphonema parvulum & $\sqrt{ }$ & $\sqrt{ }$ & $\sqrt{ }$ & $\sqrt{ }$ & $\sqrt{ }$ & $\sqrt{ }$ & $\sqrt{ }$ \\
\hline 39 & Gomphonema gracile & $\sqrt{ }$ & $\sqrt{ }$ & $\sqrt{ }$ & $\sqrt{ }$ & $\sqrt{ }$ & $\sqrt{ }$ & $\sqrt{ }$ \\
\hline 40 & Gomphonema subventricosum & $\sqrt{ }$ & $\sqrt{ }$ & $\sqrt{ }$ & - & - & - & - \\
\hline 41 & Gomphonema longiceps & $\sqrt{ }$ & $\sqrt{ }$ & - & $\sqrt{ }$ & $\sqrt{ }$ & - & - \\
\hline 42 & Gomphonema Clevei & $\sqrt{ }$ & $\sqrt{ }$ & $\sqrt{ }$ & - & - & - & - \\
\hline 43 & Gyrosigma attenuatum & - & $\sqrt{ }$ & $\sqrt{ }$ & - & - & - & - \\
\hline 44 & Melosira granulata & - & - & $\sqrt{ }$ & - & - & - & - \\
\hline
\end{tabular}


Table 3 (Cont.). Periphytic diatom occurrence at various stations (in alphabetical order).

\begin{tabular}{|c|c|c|c|c|c|c|c|c|}
\hline \multirow{3}{*}{ No } & \multirow{3}{*}{ Diatom species } & \multicolumn{7}{|c|}{ Stations } \\
\hline & & \multicolumn{4}{|c|}{ Linggi (s.s) River } & \multicolumn{3}{|c|}{ Kundur River } \\
\hline & & St. 1 & St. 2 & St. 3 & St. 4 & St. 9 & St. 10 & St. $11 \& 12$ \\
\hline 45 & Melosira italica & $\sqrt{ }$ & - & $\sqrt{ }$ & - & $\sqrt{ }$ & - & - \\
\hline 46 & Navicula senjoensis & $\sqrt{ }$ & $\sqrt{ }$ & $\sqrt{ }$ & - & - & $\sqrt{ }$ & - \\
\hline 47 & Navicula liboensis & $\sqrt{ }$ & $\sqrt{ }$ & $\sqrt{ }$ & - & - & - & - \\
\hline 48 & Navicula veneta & $\sqrt{ }$ & $\sqrt{ }$ & $\sqrt{ }$ & $\sqrt{ }$ & $\sqrt{ }$ & $\sqrt{ }$ & $\sqrt{ }$ \\
\hline 49 & Navicula globosa & $\sqrt{ }$ & $\sqrt{ }$ & $\sqrt{ }$ & $\sqrt{ }$ & - & $\sqrt{ }$ & $\sqrt{ }$ \\
\hline 50 & Navicula trituberculata & $\sqrt{ }$ & - & - & - & $\sqrt{ }$ & - & $\sqrt{ }$ \\
\hline 51 & Navicula antigua* & - & - & $\sqrt{ }$ & - & - & - & - \\
\hline 52 & Navicula mutica* & - & - & $\sqrt{ }$ & - & - & - & - \\
\hline 53 & Navicula pupula & - & $\sqrt{ }$ & $\sqrt{ }$ & - & - & $\sqrt{ }$ & $\sqrt{ }$ \\
\hline 54 & Navicula minima & - & - & - & - & - & - & $\sqrt{ }$ \\
\hline 55 & Navicula gastrum & $\sqrt{ }$ & - & $\sqrt{ }$ & - & - & $\sqrt{ }$ & $\sqrt{ }$ \\
\hline 56 & Navicula rhyncocephala & - & $\sqrt{ }$ & $\sqrt{ }$ & $\sqrt{ }$ & - & - & $\sqrt{ }$ \\
\hline 57 & Navicula cuspidata & - & - & - & - & - & $\sqrt{ }$ & $\sqrt{ }$ \\
\hline 58 & Navicula ambhibola & - & - & - & - & - & - & $\sqrt{ }$ \\
\hline 59 & Neidium productum* & - & - & $\sqrt{ }$ & - & - & - & - \\
\hline 60 & Neidium hitchockii & - & - & - & - & - & - & $\sqrt{ }$ \\
\hline 61 & Nitzschia palea & $\sqrt{ }$ & $\sqrt{ }$ & $\sqrt{ }$ & $\sqrt{ }$ & $\sqrt{ }$ & $\sqrt{ }$ & $\sqrt{ }$ \\
\hline 62 & Nitzschia amphioxys* & - & - & - & - & - & $\sqrt{ }$ & - \\
\hline 63 & Nitzschia sigma var. clausii & $\sqrt{ }$ & $\sqrt{ }$ & $\sqrt{ }$ & $\sqrt{ }$ & $\sqrt{ }$ & - & - \\
\hline 64 & Nitzschia stagnorum & - & - & - & $\sqrt{ }$ & - & $\sqrt{ }$ & $\sqrt{ }$ \\
\hline 65 & Nitzschia acicularis* & - & - & - & - & - & $\sqrt{ }$ & - \\
\hline 66 & Nitzshia sigma & - & - & - & - & - & $\sqrt{ }$ & $\sqrt{ }$ \\
\hline 67 & Nitzschia obtusa & - & - & - & - & $\sqrt{ }$ & - & $\sqrt{ }$ \\
\hline 68 & Nitzschia fonticola & $\sqrt{ }$ & - & - & $\sqrt{ }$ & - & - & - \\
\hline 69 & Pinnularia biceps & $\sqrt{ }$ & $\sqrt{ }$ & $\sqrt{ }$ & $\sqrt{ }$ & $\sqrt{ }$ & $\sqrt{ }$ & $\sqrt{ }$ \\
\hline 70 & Pinnularia Braunii & - & $\sqrt{ }$ & $\sqrt{ }$ & $\sqrt{ }$ & $\sqrt{ }$ & $\sqrt{ }$ & $\sqrt{ }$ \\
\hline 71 & Pinnularia microstauron & $\sqrt{ }$ & $\sqrt{ }$ & $\sqrt{ }$ & $\sqrt{ }$ & $\sqrt{ }$ & $\sqrt{ }$ & $\sqrt{ }$ \\
\hline 72 & Pinnularia microstauron var. ambigua & - & $\sqrt{ }$ & - & - & - & - & - \\
\hline 73 & Pinnularia bogotensis & - & $\sqrt{ }$ & - & - & $\sqrt{ }$ & - & - \\
\hline 74 & Pinnularia borealis & - & - & - & - & $\sqrt{ }$ & $\sqrt{ }$ & $\sqrt{ }$ \\
\hline 75 & Pinnularia brevicostata & - & - & $\sqrt{ }$ & - & - & - & - \\
\hline 76 & Pinnularia stauroptera & - & - & $\sqrt{ }$ & - & $\sqrt{ }$ & - & $\sqrt{ }$ \\
\hline 77 & Rhopalodia gibberula & $\sqrt{ }$ & $\sqrt{ }$ & - & - & - & - & - \\
\hline 78 & Synedra rumpens & $\sqrt{ }$ & $\sqrt{ }$ & $\sqrt{ }$ & - & - & - & - \\
\hline 79 & Surirella tenuissima & $\sqrt{ }$ & $\sqrt{ }$ & $\sqrt{ }$ & $\sqrt{ }$ & - & $\sqrt{ }$ & $\sqrt{ }$ \\
\hline 80 & Surirella angusta & $\sqrt{ }$ & $\sqrt{ }$ & - & - & - & $\sqrt{ }$ & $\sqrt{ }$ \\
\hline 81 & Surirella linearis & $\sqrt{ }$ & - & $\sqrt{ }$ & - & - & - & - \\
\hline 82 & Surirella lemmermanii & $\sqrt{ }$ & $\sqrt{ }$ & - & - & - & - & - \\
\hline 83 & Surirella robusta & - & $\sqrt{ }$ & $\sqrt{ }$ & - & - & - & - \\
\hline 84 & Surirella angusticostata* & - & - & - & - & - & $\sqrt{ }$ & - \\
\hline 85 & Stauroneis pusilla & $\sqrt{ }$ & - & $\sqrt{ }$ & - & - & - & $\sqrt{ }$ \\
\hline \multirow[t]{2}{*}{86} & Stauroneis phoenicenteron & $\sqrt{ }$ & - & - & - & - & - & $\sqrt{ }$ \\
\hline & Total & 48 & 45 & 46 & 26 & 23 & 28 & 34 \\
\hline
\end{tabular}


Table 4. Periphytic diatom occurrence at various stations based on constancy values.

\begin{tabular}{|c|c|c|c|c|c|c|c|c|c|}
\hline \multirow{3}{*}{ No } & \multirow{3}{*}{ Diatom Species } & \multicolumn{8}{|c|}{ Stations } \\
\hline & & \multicolumn{4}{|c|}{ Linggi River } & \multicolumn{4}{|c|}{ Kundur River } \\
\hline & & St. 1 & St. 2 & St. 3 & St. 4 & St. 9 & St. 10 & St. $11 \& 12$ & All \\
\hline 1 & Achnanthes minutissima & $\sqrt{ }$ & $\sqrt{ }$ & $\sqrt{ }$ & $\sqrt{ }$ & $\sqrt{ }$ & $\sqrt{ }$ & $\sqrt{ }$ & 7 \\
\hline 2 & Eunotia monodon & $\sqrt{ }$ & $\sqrt{ }$ & $\sqrt{ }$ & $\sqrt{ }$ & $\sqrt{ }$ & $\sqrt{ }$ & $\sqrt{ }$ & 7 \\
\hline 3 & Frustulia saxonica & $\sqrt{ }$ & $\sqrt{ }$ & $\sqrt{ }$ & $\sqrt{ }$ & $\sqrt{ }$ & $\sqrt{ }$ & $\sqrt{ }$ & 7 \\
\hline 4 & Gophonema parvulum & $\sqrt{ }$ & $\sqrt{ }$ & $\sqrt{ }$ & $\sqrt{ }$ & $\sqrt{ }$ & $\sqrt{ }$ & $\sqrt{ }$ & 7 \\
\hline 5 & Gophonema gracile & $\sqrt{ }$ & $\sqrt{ }$ & $\sqrt{ }$ & $\sqrt{ }$ & $\sqrt{ }$ & $\sqrt{ }$ & $\sqrt{ }$ & 7 \\
\hline 6 & Navicula veneta & $\sqrt{ }$ & $\sqrt{ }$ & $\sqrt{ }$ & $\sqrt{ }$ & $\sqrt{ }$ & $\sqrt{ }$ & $\sqrt{ }$ & 7 \\
\hline 7 & Nitzschia palea & $\sqrt{ }$ & $\sqrt{ }$ & $\sqrt{ }$ & $\sqrt{ }$ & $\sqrt{ }$ & $\sqrt{ }$ & $\sqrt{ }$ & 7 \\
\hline 8 & Pinnularia microstauron & $\sqrt{ }$ & $\sqrt{ }$ & $\sqrt{ }$ & $\sqrt{ }$ & $\sqrt{ }$ & $\sqrt{ }$ & $\sqrt{ }$ & 7 \\
\hline 9 & Pinnularia biceps & $\sqrt{ }$ & $\sqrt{ }$ & $\sqrt{ }$ & $\sqrt{ }$ & $\sqrt{ }$ & $\sqrt{ }$ & $\sqrt{ }$ & 7 \\
\hline 10 & Achnanthes linearis & $\sqrt{ }$ & $\sqrt{ }$ & $\sqrt{ }$ & $\sqrt{ }$ & $\sqrt{ }$ & $\sqrt{ }$ & - & 6 \\
\hline 11 & Achnanthes saxonica & $\sqrt{ }$ & $\sqrt{ }$ & $\sqrt{ }$ & $\sqrt{ }$ & $\sqrt{ }$ & $\sqrt{ }$ & - & 6 \\
\hline 12 & Cymbella javanica & $\sqrt{ }$ & $\sqrt{ }$ & $\sqrt{ }$ & $\sqrt{ }$ & $\sqrt{ }$ & - & $\sqrt{ }$ & 6 \\
\hline 13 & Navicula globosa & $\sqrt{ }$ & $\sqrt{ }$ & $\sqrt{ }$ & $\sqrt{ }$ & - & $\sqrt{ }$ & $\sqrt{ }$ & 6 \\
\hline 14 & Pinnularia Braunii & - & $\sqrt{ }$ & $\sqrt{ }$ & $\sqrt{ }$ & $\sqrt{ }$ & $\sqrt{ }$ & $\sqrt{ }$ & 6 \\
\hline 15 & Surirella tenuissima & $\sqrt{ }$ & $\sqrt{ }$ & $\sqrt{ }$ & $\sqrt{ }$ & - & $\sqrt{ }$ & $\sqrt{ }$ & 6 \\
\hline 16 & Achnanthes exigua & $\sqrt{ }$ & $\sqrt{ }$ & - & $\sqrt{ }$ & - & $\sqrt{ }$ & $\sqrt{ }$ & 5 \\
\hline 17 & Nitzschia sigma var. clausii & $\sqrt{ }$ & $\sqrt{ }$ & $\sqrt{ }$ & $\sqrt{ }$ & $\sqrt{ }$ & - & - & 5 \\
\hline 18 & Cyclotella meneghiniana & - & $\sqrt{ }$ & $\sqrt{ }$ & $\sqrt{ }$ & - & - & $\sqrt{ }$ & 4 \\
\hline 19 & Cymbella turgida & $\sqrt{ }$ & $\sqrt{ }$ & $\sqrt{ }$ & - & - & - & $\sqrt{ }$ & 4 \\
\hline 20 & Eunotia major & - & $\sqrt{ }$ & $\sqrt{ }$ & $\sqrt{ }$ & $\sqrt{ }$ & - & - & 4 \\
\hline 21 & Gomphonema longiceps & $\sqrt{ }$ & $\sqrt{ }$ & - & $\sqrt{ }$ & $\sqrt{ }$ & - & - & 4 \\
\hline 22 & Navicula gastrum & $\sqrt{ }$ & - & $\sqrt{ }$ & - & - & $\sqrt{ }$ & $\sqrt{ }$ & 4 \\
\hline 23 & Navicula rhyncocephala & - & $\sqrt{ }$ & $\sqrt{ }$ & $\sqrt{ }$ & - & - & $\sqrt{ }$ & 4 \\
\hline 24 & Navicula senjoensis & $\sqrt{ }$ & $\sqrt{ }$ & $\sqrt{ }$ & - & - & $\sqrt{ }$ & - & 4 \\
\hline 25 & Navicula pupula & - & $\sqrt{ }$ & $\sqrt{ }$ & - & - & $\sqrt{ }$ & $\sqrt{ }$ & 4 \\
\hline 26 & Surirella angusta & $\sqrt{ }$ & $\sqrt{ }$ & - & - & - & $\sqrt{ }$ & $\sqrt{ }$ & 4 \\
\hline 27 & Achnanthes brevipes & $\sqrt{ }$ & $\sqrt{ }$ & $\sqrt{ }$ & - & - & - & - & 3 \\
\hline 28 & Amphora normani & $\sqrt{ }$ & - & $\sqrt{ }$ & $\sqrt{ }$ & - & - & - & 3 \\
\hline 29 & Cymbella sumatrensis & $\sqrt{ }$ & $\sqrt{ }$ & $\sqrt{ }$ & - & - & - & - & 3 \\
\hline 30 & Cymbella kolbei & $\sqrt{ }$ & - & $\sqrt{ }$ & - & - & $\sqrt{ }$ & - & 3 \\
\hline 31 & Gomphonema Clevi & $\sqrt{ }$ & $\sqrt{ }$ & $\sqrt{ }$ & - & - & - & - & 3 \\
\hline 32 & Gomphonema subventricosum & $\sqrt{ }$ & $\sqrt{ }$ & $\sqrt{ }$ & - & - & - & - & 3 \\
\hline 33 & Melosira italica & $\sqrt{ }$ & - & $\sqrt{ }$ & - & $\sqrt{ }$ & - & - & 3 \\
\hline 34 & Navicula liboensis & $\sqrt{ }$ & $\sqrt{ }$ & $\sqrt{ }$ & - & - & - & - & 3 \\
\hline 35 & Navicula trituberculata & $\sqrt{ }$ & - & - & - & $\sqrt{ }$ & - & $\sqrt{ }$ & 3 \\
\hline 36 & Nitzschia stagnorum & - & - & - & $\sqrt{ }$ & - & $\sqrt{ }$ & $\sqrt{ }$ & 3 \\
\hline 37 & Pinnularia borealis & - & - & - & - & $\sqrt{ }$ & $\sqrt{ }$ & $\sqrt{ }$ & 3 \\
\hline 38 & Pinnularia stauroptera & - & - & $\sqrt{ }$ & - & $\sqrt{ }$ & - & $\sqrt{ }$ & 3 \\
\hline 39 & Synedra rumpens & $\sqrt{ }$ & $\sqrt{ }$ & $\sqrt{ }$ & - & - & - & - & 3 \\
\hline 40 & Stauroneis pusilla & $\sqrt{ }$ & - & $\sqrt{ }$ & - & - & - & $\sqrt{ }$ & 3 \\
\hline 41 & Achnanthes lapidosa & $\sqrt{ }$ & $\sqrt{ }$ & - & - & - & - & - & 2 \\
\hline 42 & Achnanthes stauroneiformis & $\sqrt{ }$ & - & $\sqrt{ }$ & - & - & - & - & 2 \\
\hline 43 & Cyclotella glomerata & $\sqrt{ }$ & $\sqrt{ }$ & - & - & - & - & - & 2 \\
\hline 44 & Cymbella minuta & $\sqrt{ }$ & $\sqrt{ }$ & - & - & - & - & - & 2 \\
\hline
\end{tabular}


Table 4 (Cont.). Periphytic diatom occurrence at various stations based on constancy values.

\begin{tabular}{|c|c|c|c|c|c|c|c|c|c|}
\hline \multirow{3}{*}{ No } & \multirow{3}{*}{ Diatom Species } & \multicolumn{8}{|c|}{ Stations } \\
\hline & & \multicolumn{4}{|c|}{ Linggi River } & \multicolumn{4}{|c|}{ Kundur River } \\
\hline & & St. 1 & St. 2 & St. 3 & St. 4 & St. 9 & St. 10 & St. $11 \& 12$ & All \\
\hline 45 & Cymbella sumatrensis fo. malayensis & $\sqrt{ }$ & - & - & $\sqrt{ }$ & - & - & - & 2 \\
\hline 46 & Diploneis ovalis & $\sqrt{ }$ & - & $\sqrt{ }$ & - & - & - & - & 2 \\
\hline 47 & Gyrosigma attenuatum & - & $\sqrt{ }$ & $\sqrt{ }$ & - & - & - & - & 2 \\
\hline 48 & Navicula cuspidata & - & - & - & - & - & $\sqrt{ }$ & $\sqrt{ }$ & 2 \\
\hline 49 & Nitzschia fonticola & $\sqrt{ }$ & - & - & $\sqrt{ }$ & - & - & - & 2 \\
\hline 50 & Nitzschia obtusa & - & - & - & - & $\sqrt{ }$ & - & $\sqrt{ }$ & 2 \\
\hline 51 & Nitzschia sigma & - & - & - & - & - & $\sqrt{ }$ & $\sqrt{ }$ & 2 \\
\hline 52 & Pinnularia Bogotensis & - & $\sqrt{ }$ & - & - & $\sqrt{ }$ & - & - & 2 \\
\hline 53 & Rhopalodia gibberula & $\sqrt{ }$ & $\sqrt{ }$ & - & - & - & - & - & 2 \\
\hline 54 & Surirella linearis & $\sqrt{ }$ & - & $\sqrt{ }$ & - & - & - & - & 2 \\
\hline 55 & Surirella lemmermanii & $\sqrt{ }$ & $\sqrt{ }$ & - & - & - & - & - & 2 \\
\hline 56 & Surirella robusta & - & $\sqrt{ }$ & $\sqrt{ }$ & - & - & - & - & 2 \\
\hline 57 & Stauroneis phoenicenteron & $\sqrt{ }$ & - & - & - & - & - & $\sqrt{ }$ & 2 \\
\hline 58 & Achnanthes crenulata & $\sqrt{ }$ & - & - & - & - & - & - & 1 \\
\hline 59 & Cymbella cuspidata & - & - & - & $\sqrt{ }$ & - & - & - & 1 \\
\hline 60 & Cymbella japonica & $\sqrt{ }$ & - & - & - & - & - & - & 1 \\
\hline 61 & Cymbella tumida & $\sqrt{ }$ & - & - & - & - & - & - & 1 \\
\hline 62 & Cocononeis thumensis & - & $\sqrt{ }$ & - & - & - & - & - & 1 \\
\hline 63 & Desmogonium rabenhorstianum & - & $\sqrt{ }$ & - & - & - & - & - & 1 \\
\hline 64 & Eunotia camelus & - & - & - & - & - & $\sqrt{ }$ & - & 1 \\
\hline 65 & Eunotia lunaris & - & - & - & - & $\sqrt{ }$ & - & - & 1 \\
\hline 66 & Eunotia major van indica & - & - & - & - & - & - & $\sqrt{ }$ & 1 \\
\hline 67 & Eunotia polydentula & - & - & $\sqrt{ }$ & - & - & - & - & 1 \\
\hline 68 & Eunotia pectinalis & - & $\sqrt{ }$ & - & - & - & - & - & 1 \\
\hline 69 & Eunotia rhomboidea & - & $\sqrt{ }$ & - & - & - & - & - & 1 \\
\hline 70 & Eunotia vanheurckii & - & - & - & - & - & - & $\sqrt{ }$ & 1 \\
\hline 71 & Fragilaria sp. & $\sqrt{ }$ & - & - & - & - & - & - & 1 \\
\hline 72 & Fragilaria vaucheriae & $\sqrt{ }$ & - & - & - & - & - & - & 1 \\
\hline 73 & Frustulia rhomboides & - & - & $\sqrt{ }$ & - & - & - & - & 1 \\
\hline 74 & Frustulia javanica & - & $\sqrt{ }$ & - & - & - & - & - & 1 \\
\hline 75 & Melosira granulata & - & - & $\sqrt{ }$ & - & - & - & - & 1 \\
\hline 76 & Navicula Antigua & - & - & $\sqrt{ }$ & - & - & - & - & 1 \\
\hline 77 & Navicula mutica & - & - & $\sqrt{ }$ & - & - & - & - & 1 \\
\hline 78 & Navicula minima & - & - & - & - & - & - & $\sqrt{ }$ & 1 \\
\hline 79 & Navicula ambhibola & - & - & - & - & - & - & $\sqrt{ }$ & 1 \\
\hline 80 & Neidium hitchockii & - & - & - & - & - & - & $\sqrt{ }$ & 1 \\
\hline 81 & Neidium productum & - & - & $\sqrt{ }$ & - & - & - & - & 1 \\
\hline 82 & Nitzschia acicularis & - & - & - & - & - & $\sqrt{ }$ & - & 1 \\
\hline 83 & Nitzschia amphioxys & - & - & - & - & - & $\sqrt{ }$ & - & 1 \\
\hline 84 & Pinnularia microstauron van. ambigua & - & $\sqrt{ }$ & - & - & - & - & - & 1 \\
\hline 85 & Pinnularia brevicostata & - & - & $\sqrt{ }$ & - & - & - & - & 1 \\
\hline \multirow[t]{2}{*}{86} & Surirella angusticostata & - & - & - & - & - & $\sqrt{ }$ & - & 1 \\
\hline & Total & 48 & 45 & 46 & 26 & 23 & 28 & 34 & \\
\hline
\end{tabular}


1 were Achnanthes linearis, C. javanica, Cymbella turgida, Gomphonema parvulum and S. rumpens and also showed $100 \%$ constancy values. Most of the above-mentioned diatoms were commonly found in clean and rocky streams.

A. saxonica was the most dominant species (59\%) at Station 2, followed by Eunotia vanheurckii $(22 \%)$, they together constituted over $81 \%$ of total cell count. Except for these two species, all other species at this station showed less than $100 \%$ constancy values. A. minutissima, the second most dominant species at Station 1, constituted only $25 \%$ of cell count with a $75 \%$ constancy value here at Station 2. The lower abundance of A. minutissima, greater abundance of E. vanheurckii and appearance of new species Gyrosigma attenuatum differentiated Station 2 from Station1, though both the stations were more or less similar in water quality.

At Station 3, E. vanheurckii, A. saxonica and $A$. minutissima were the dominant species with percentage abundance of $29 \%, 20 \%$ and $11.4 \%$, respectively. A. saxonica, the second most abundant species with constancy value of $92 \%$ while the other two species showed $100 \%$ constancy values. In addition, four more species namely C. javanica, G. parvulum, Navicula cryptocephala and Pinnularia braunii, also showed $100 \%$ constancy values and contributed significantly to the total percentage abundance with $4 \%, 7 \%, 6.4 \%$ and $4 \%$, respectively. Thus, unlike the previous two stations, the Station 3 was not dominated by individuals of one or two species but many species.

At Station 4, Nitzschia palea, G. parvulum and $P$. braunii were the most dominant species with percentage abundance of $43 \%, 37 \%$ and $7.2 \%$, respectively. These three species showed $100 \%$ constancy values at Station 4 . Other common diatoms recorded at this station were Navicula globosa, Navicula gastrum, Navicula senjoensis and Pinnularia biceps.
E. vanheurckii was the most dominant species at all three stations of Kundor river $(40 \%, 33.4 \%$ and $44.2 \%$ at Station 9, 10 and 11 , respectively), followed by $N$. palea $(28 \%$, $32 \%$ and $38 \%$ respectively) and Gomphonema parvulum (18\%, 20\% and $2.4 \%$, respectively). Among them only N. palea showed 100\% constancy values at all the four stations. $P$. braunii which showed $100 \%$ constancy values at these stations, but not in abundant in numbers $(3.2 \%, 7.0 \%$, less than $1 \%)$. All the species mentioned above were pollution tolerant species (Palmer 1969).

\section{Diversity of Periphytic Diatoms}

The most common and abundant species were A. minutissima and A. saxonica at Station 1, A. saxonica and E. vanheurckii at Station 2, N. palea, G. parvulum and P. braunii at Station 3, G. parvulum and N. palea at station 4 . The lower abundance of $A$. minutissima, greater abundance of E. vanheurckii, and the appearance of Gyrosigma sp. differentiate Station 2 from Station 1 (Tables 3 and 4). Among the four stations in the Linggi (s.s.) river, the highest diversity was recorded at Station 3 which was polluted by mainly rubber effluent followed by Station 1 and 2 which were relatively unpolluted. Lowest mean diversity was recorded at Station 4, polluted mainly with urban domestic sewage and industrial wastes.

Among stations located in the Kundor river most common and abundant species of diatoms were E. vanheurckii, Gomphonema parvulum and N. palea at station 9, E. vanheurckii, G. parvulum, and Nitzschia stagnorum at station 10 and Eunotia vanheurckii and N. palea at station 11 and 12. Among four stations at Kundor river, the highest mean diversity value was recorded at Station 11 followed by Station 12, both located at the downstream pollution recovery zone. The lowest diversity was recorded at Station 9 polluted with rubber effluent (Nather Khan 1985, 1991a; Nather Khan et al. 1986a, b). 


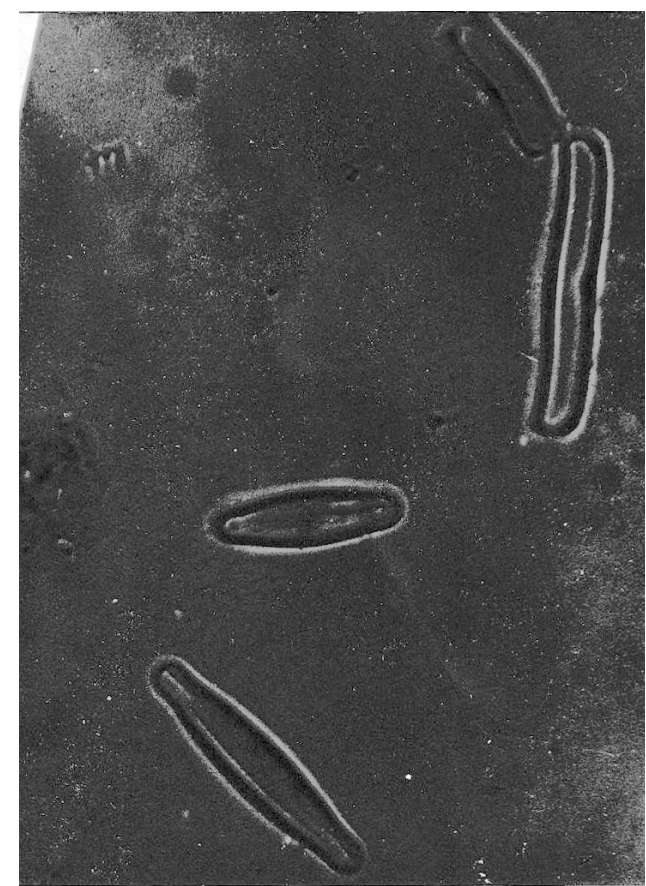

Achnanthes minutissima var. minutissima

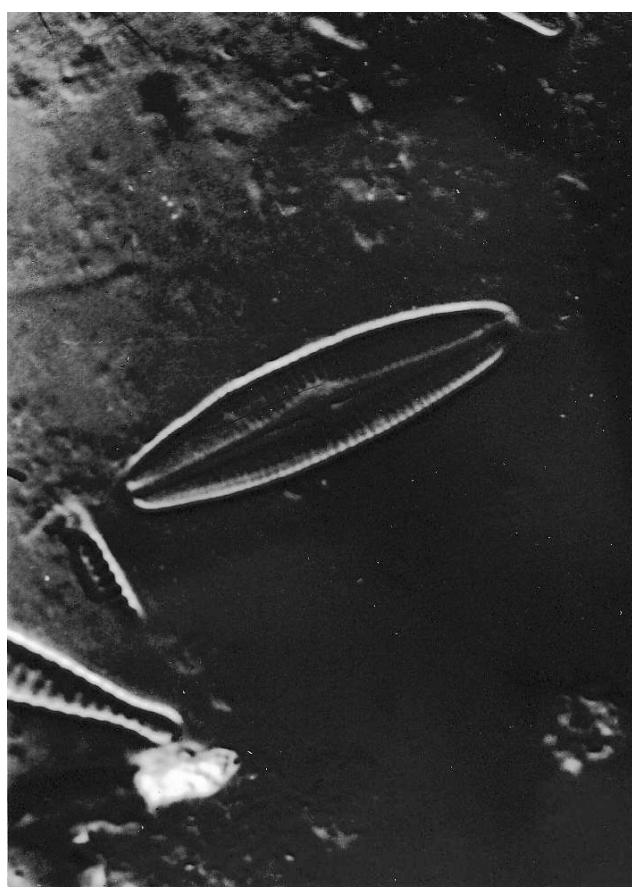

Achnanthes lapidosa var. lanceolate

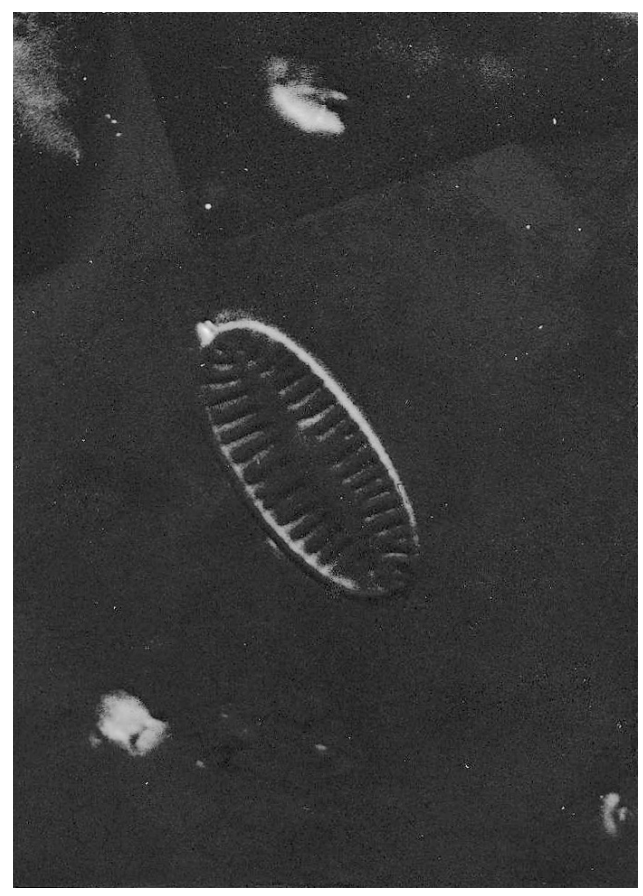

Achnanthes saxonica

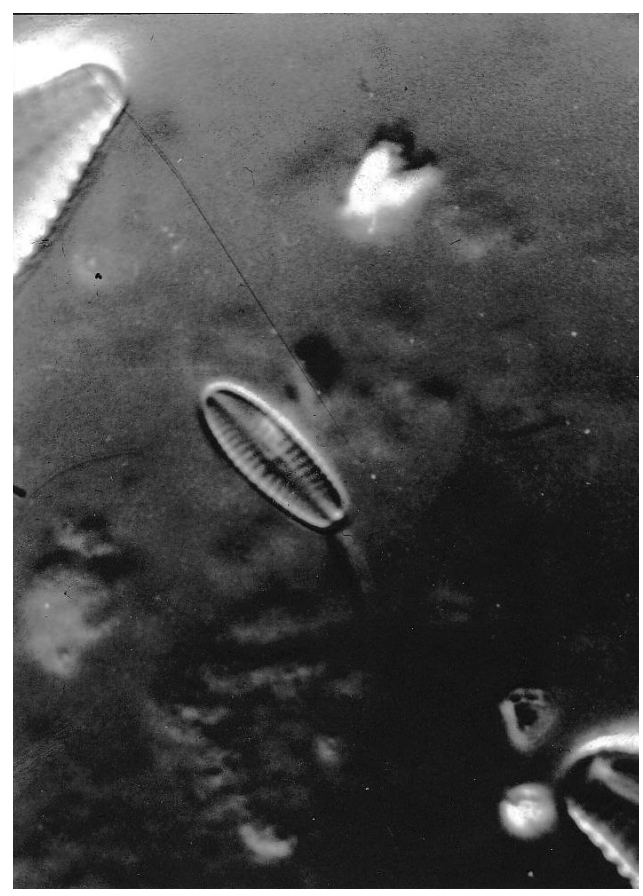

Achnanthes linearis var. linearis

Figure 2. Periphytic diatoms recorded in the Linggi (s.s.) and Kundor rivers. 


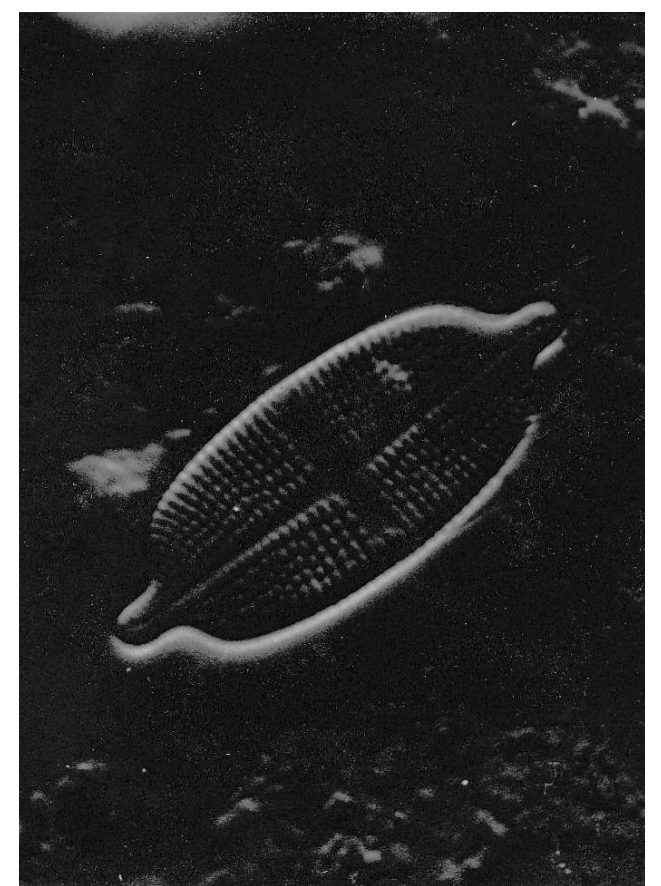

Achnanthes stauroneiformis

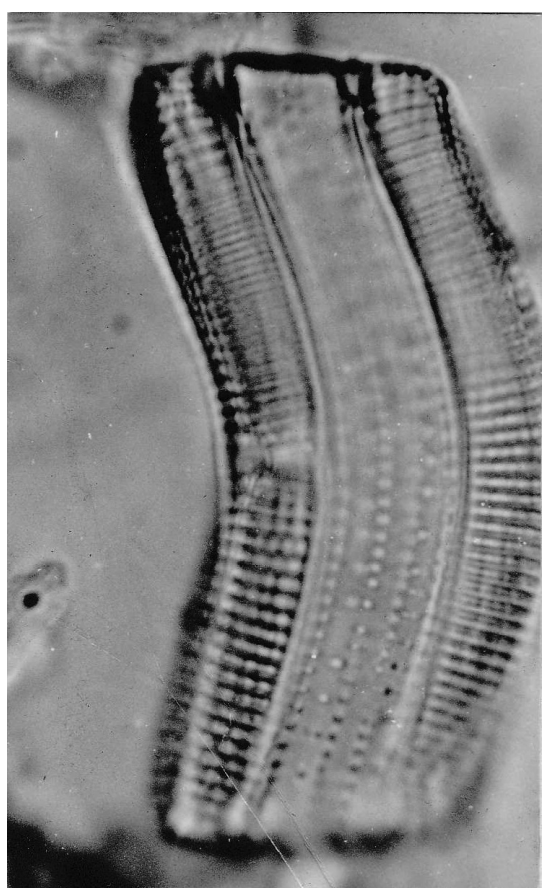

Achnanthes brevipes var. intermedia

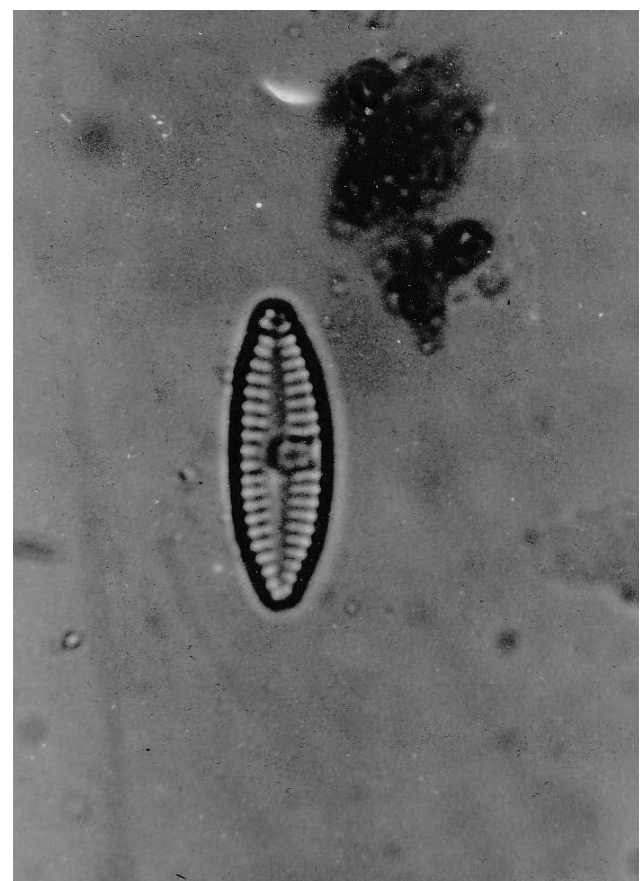

Achnanthes lapidosa var. lanceolata

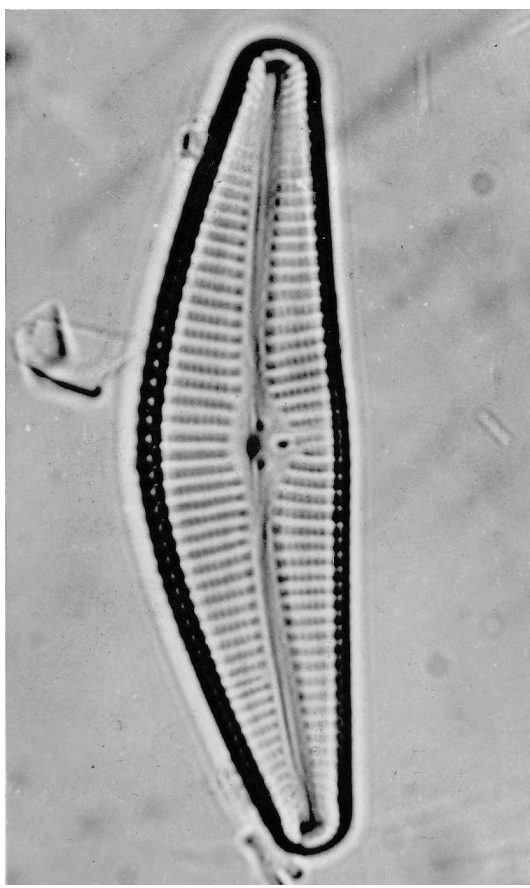

Cymbella sumatrensis fo. malayensis

Figure 2. (Cont.) Periphytic diatoms recorded in the Linggi (s.s.) and Kundor rivers. 


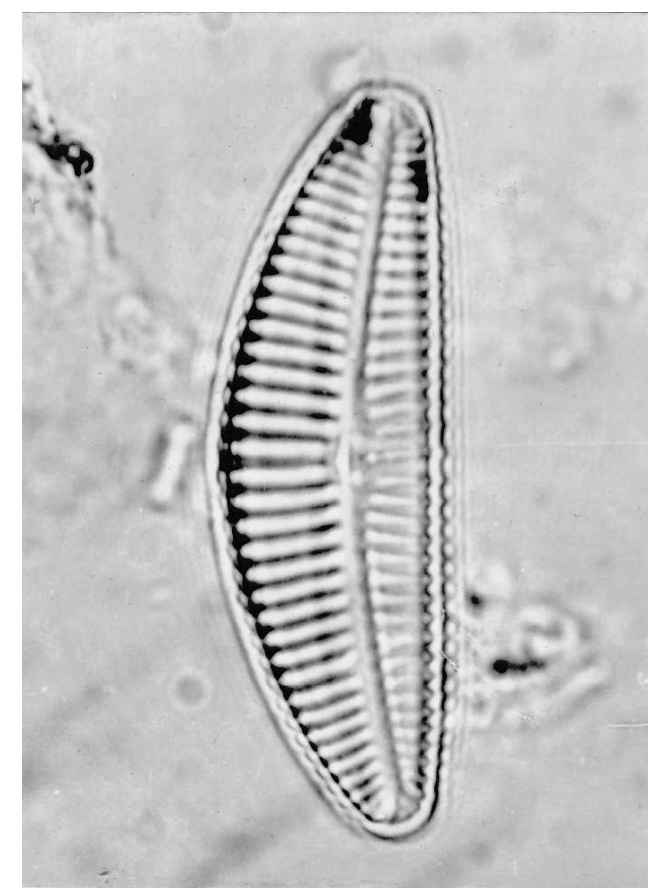

Cymbella sumatrensis

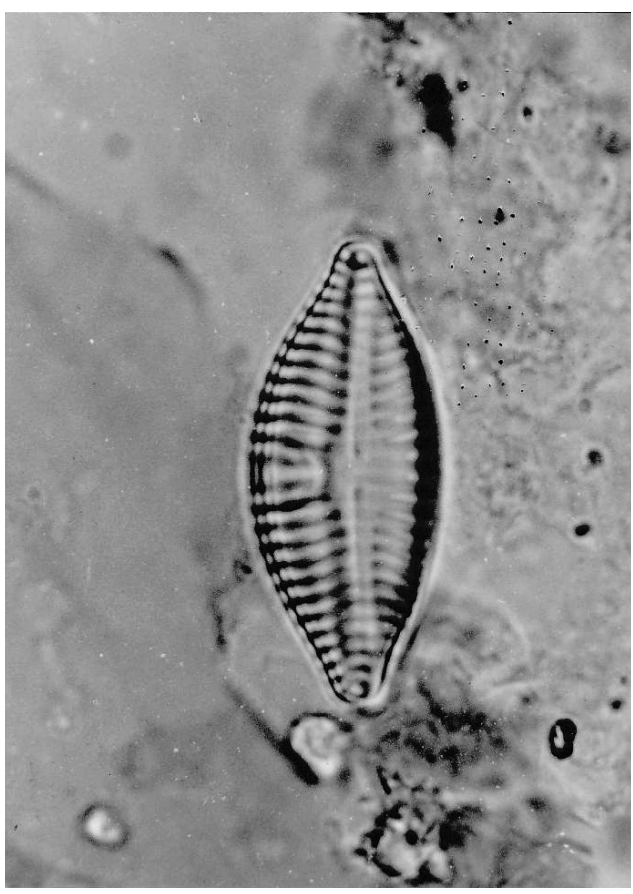

Cymbella cuspidata

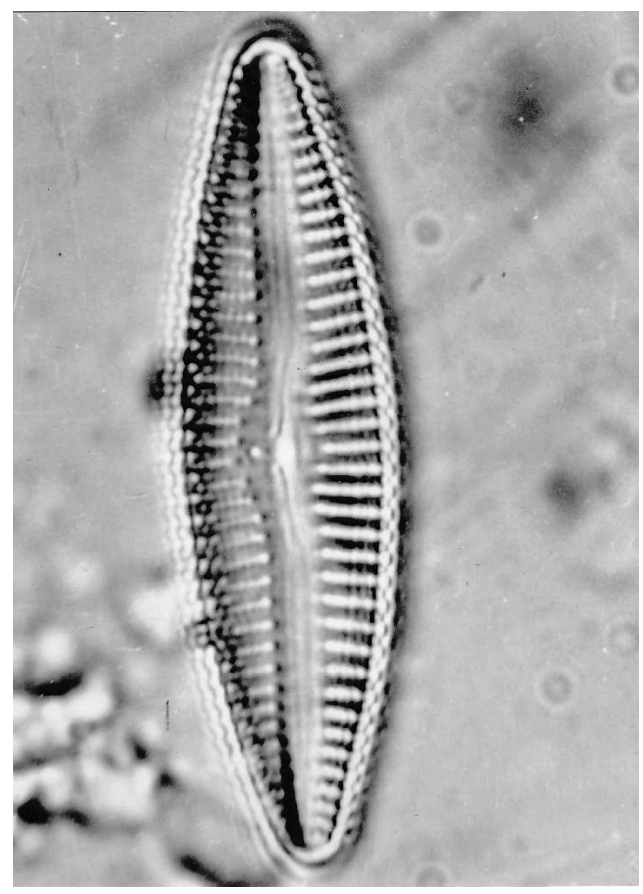

Cymbella japonica

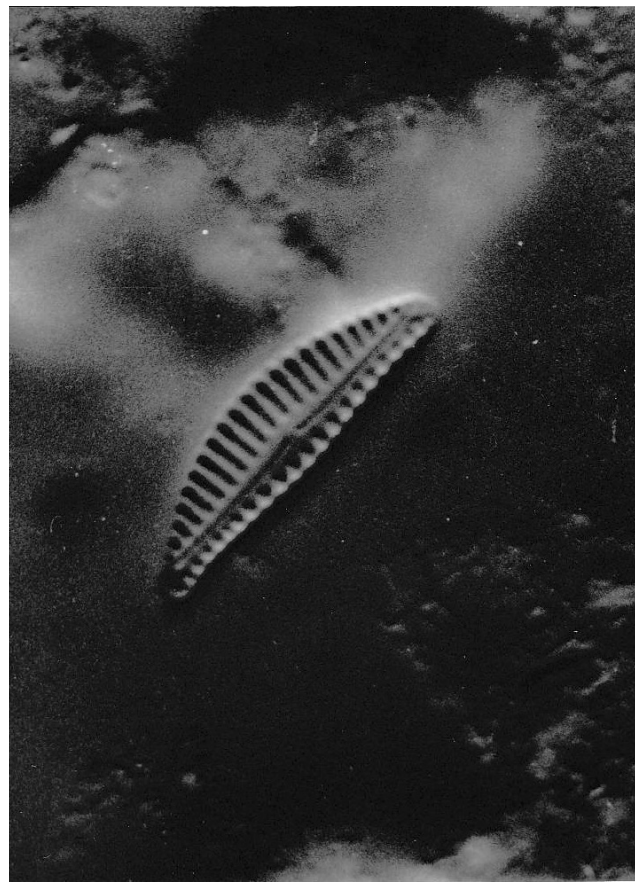

Cymbella minuta var. minuta

Figure 2. (Cont.) Periphytic diatoms recorded in the Linggi (s.s.) and Kundor rivers. 


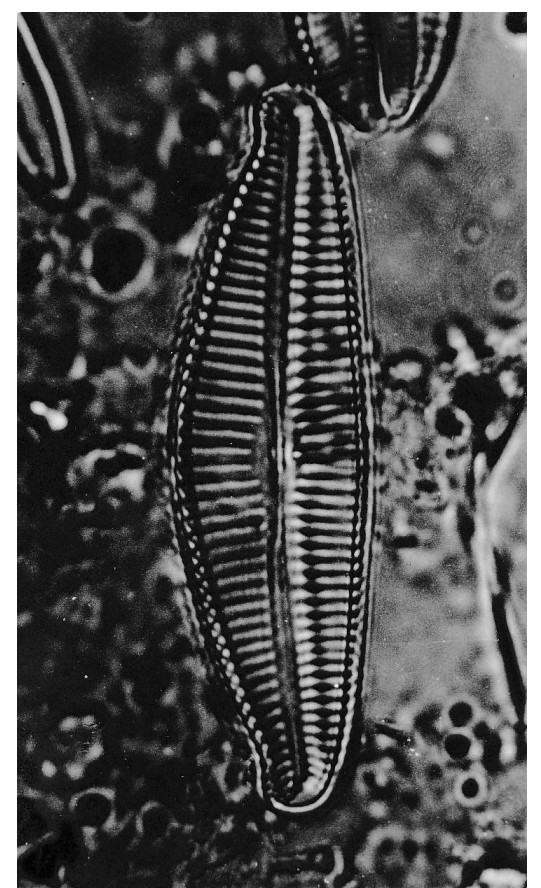

Cymbella turgida

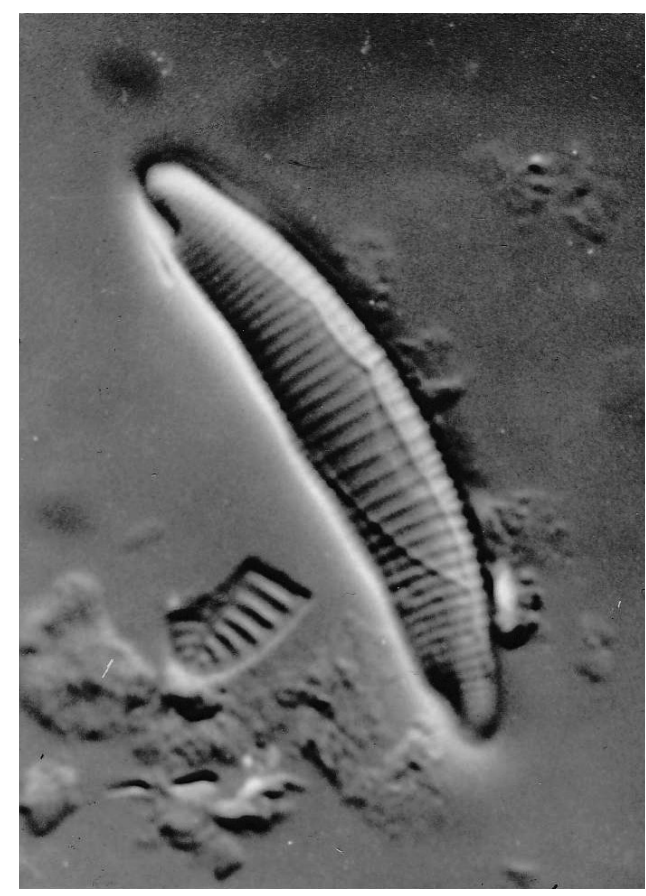

Eunotia vanheurckii

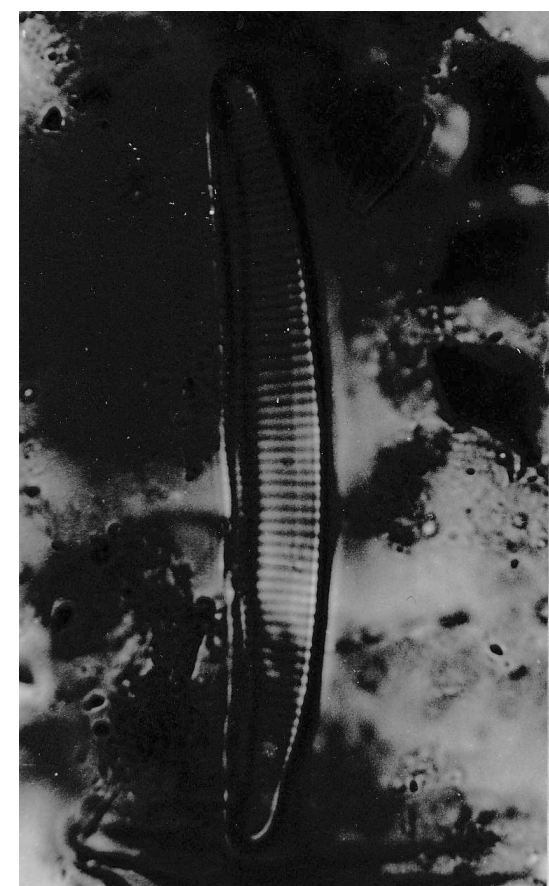

Eunotia pectinalis

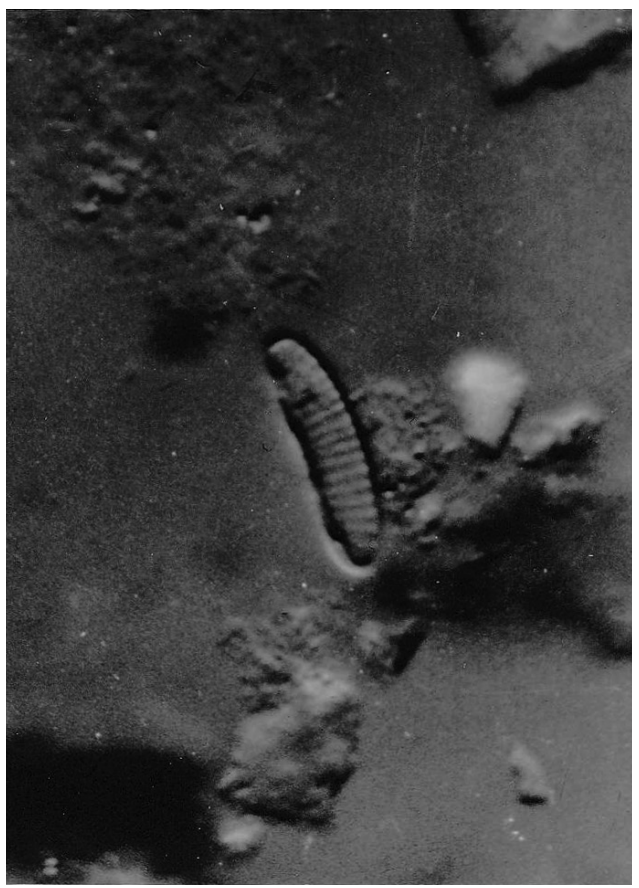

Eunotia rhomboidea

Figure 2. (Cont.) Periphytic diatoms recorded in the Linggi (s.s.) and Kundor rivers. 


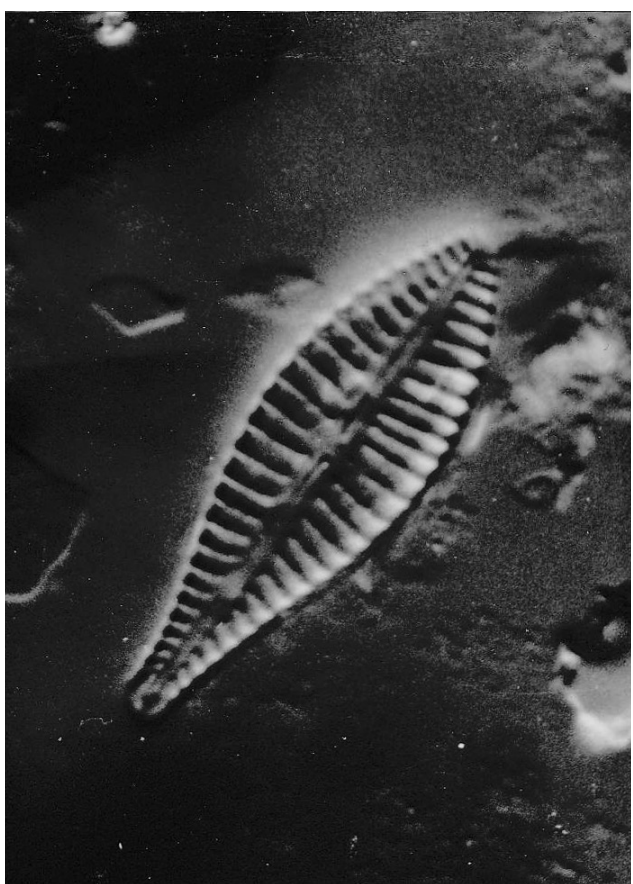

Gomphonema augustatum var. producta

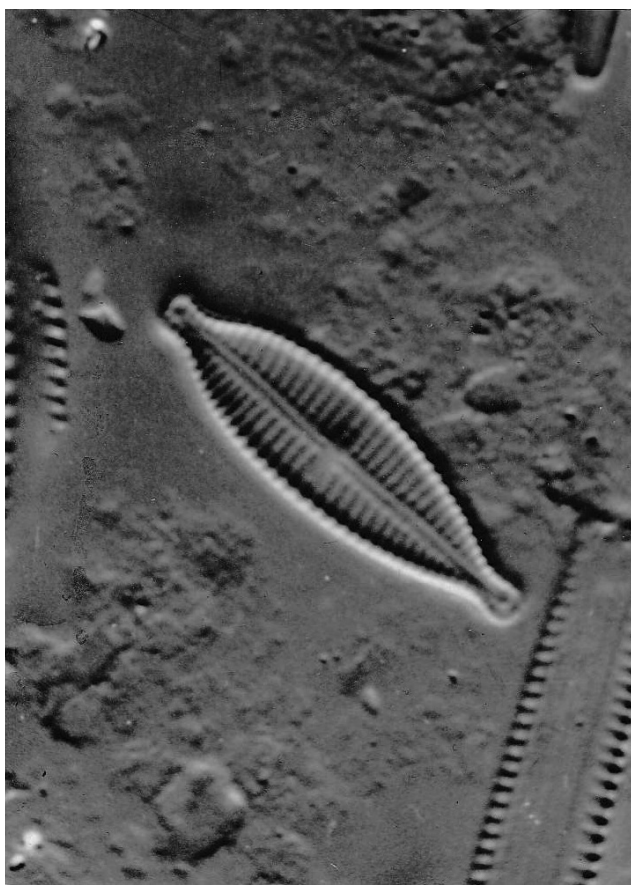

Gomphonema parvulum

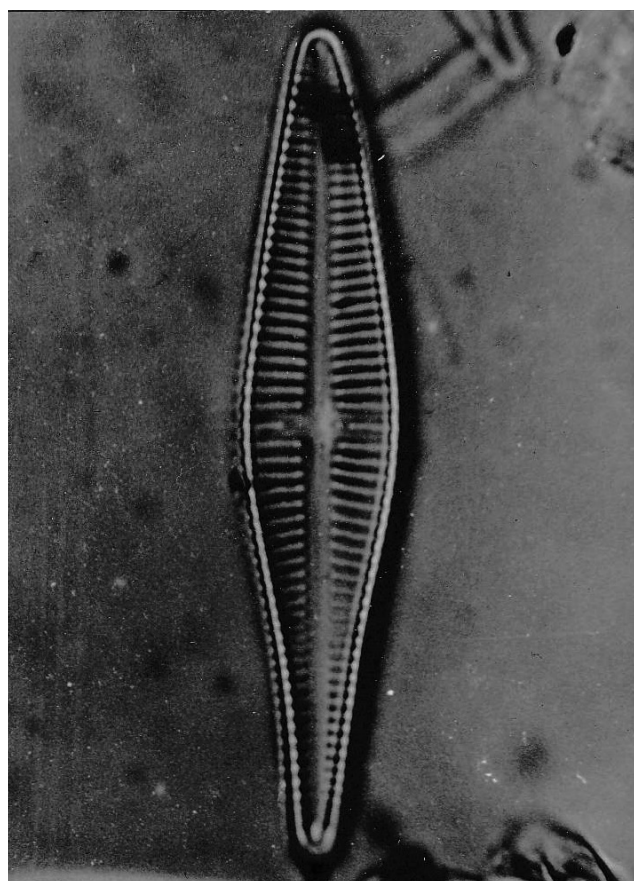

Gomphonema longiceps

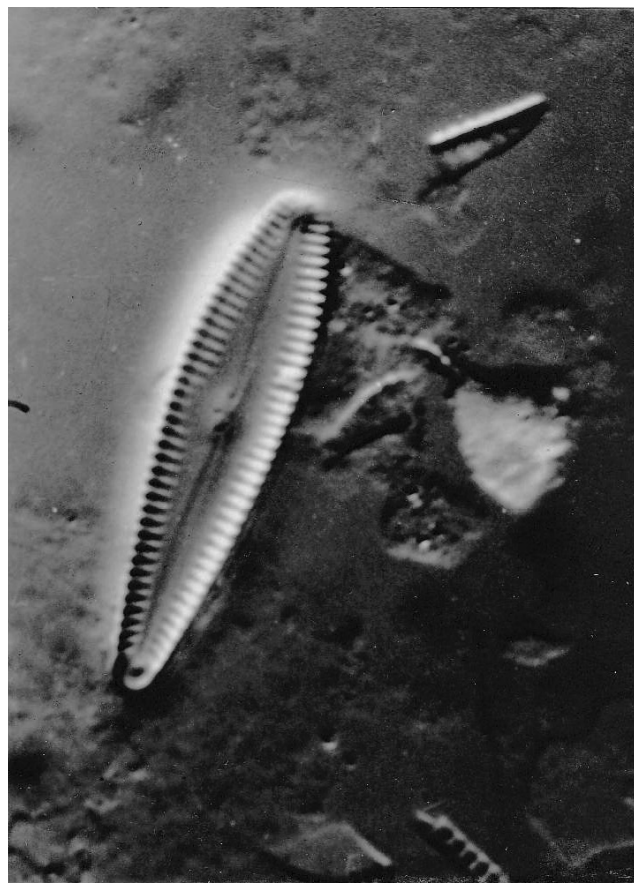

Gomphonema clevei var. inaequilongum

Figure 2. (Cont.) Periphytic diatoms recorded in the Linggi (s.s.) and Kundor rivers. 


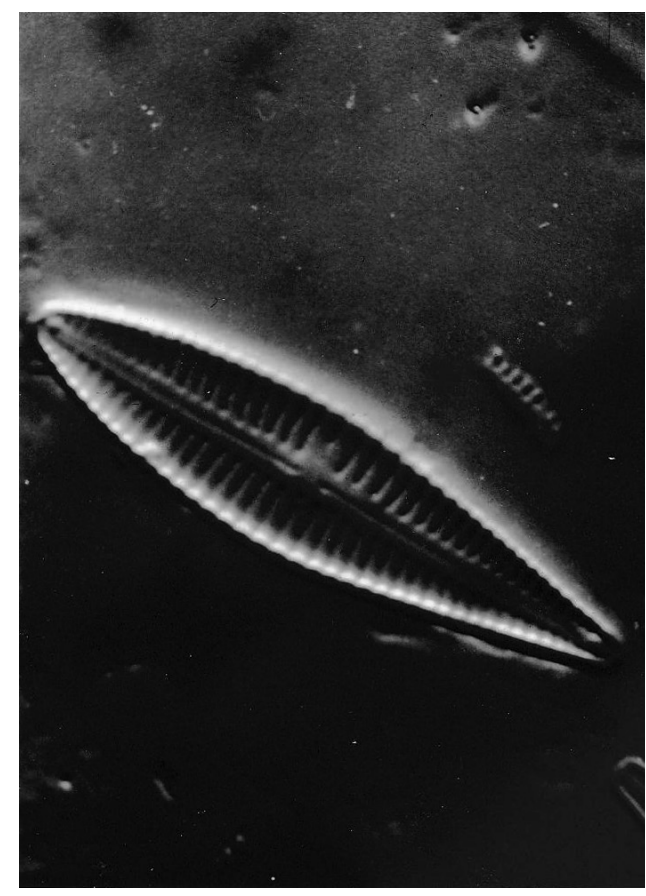

Gomphonema gracile

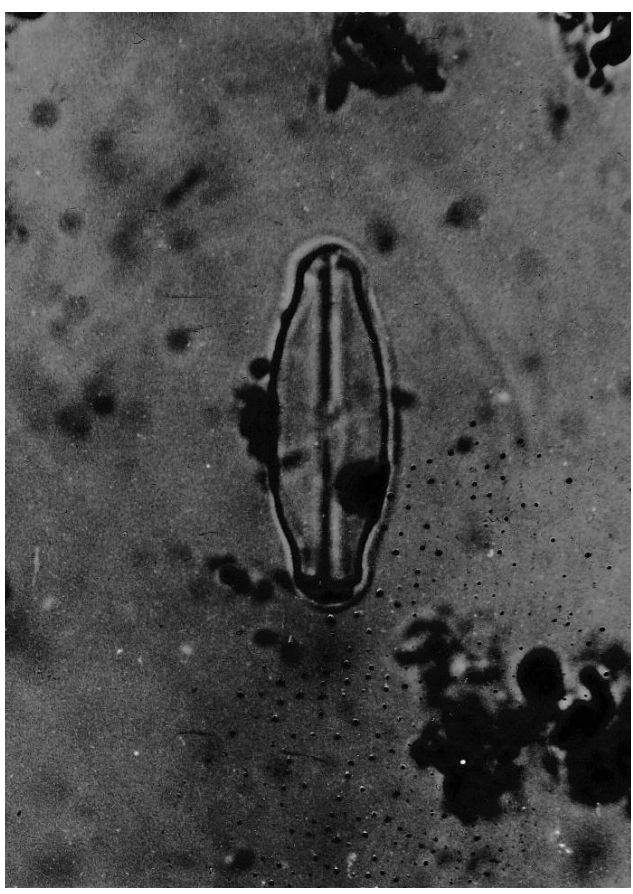

Navicula amphibola

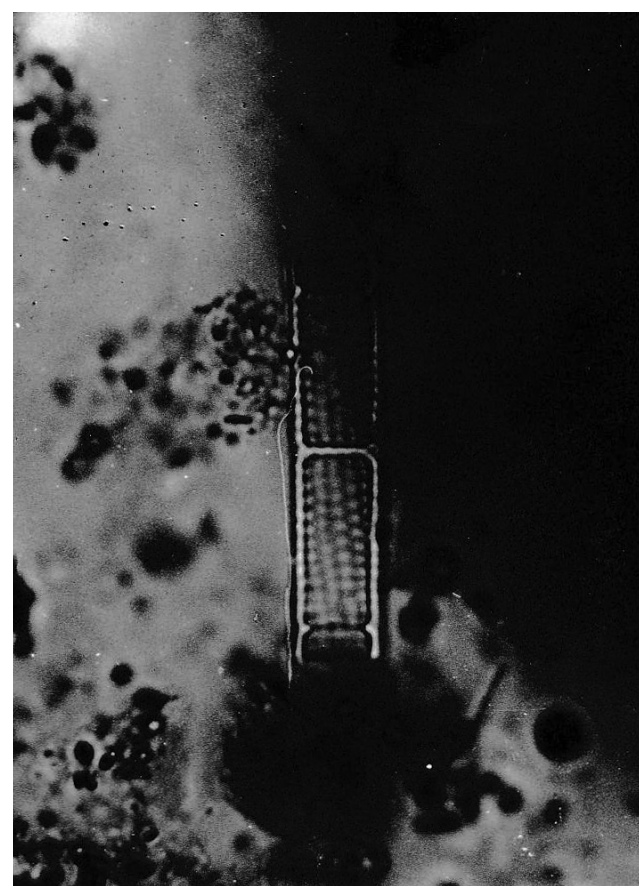

Melosira granulata

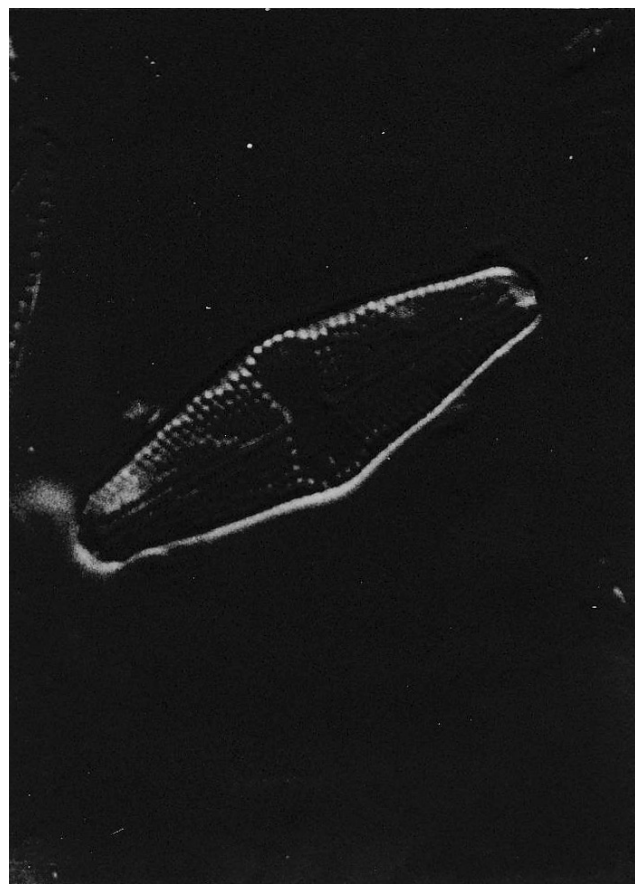

Navicula mutica fo. intermedia

Figure 2. (Cont.) Periphytic diatoms recorded in the Linggi (s.s.) and Kundor rivers. 


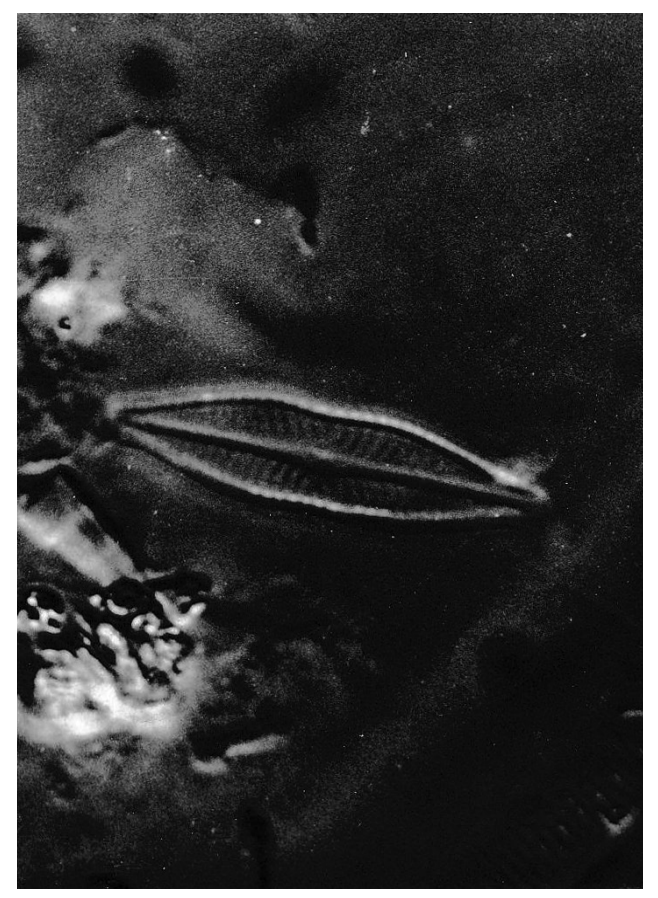

Navicula pavillardi

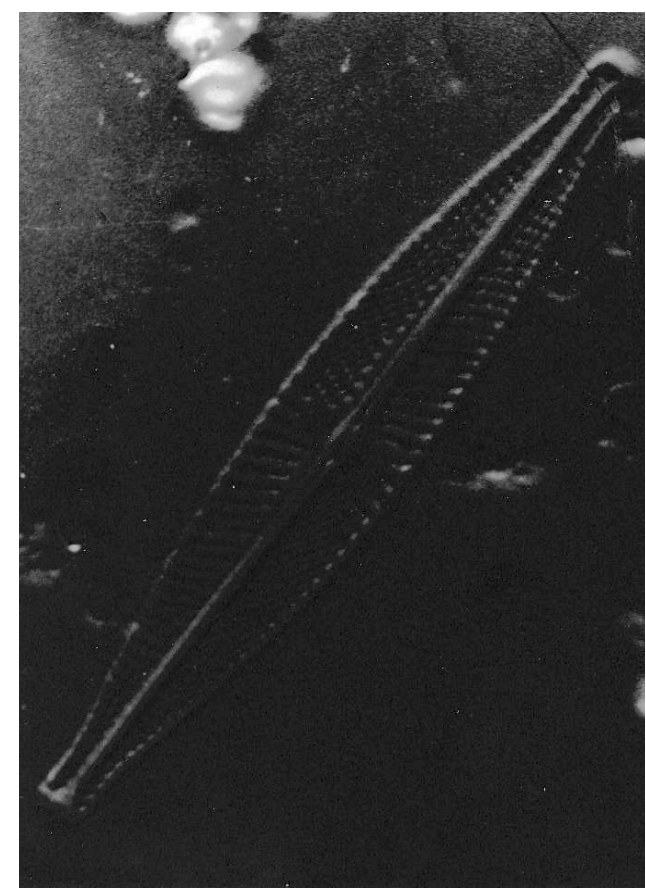

Navicula senjoensis

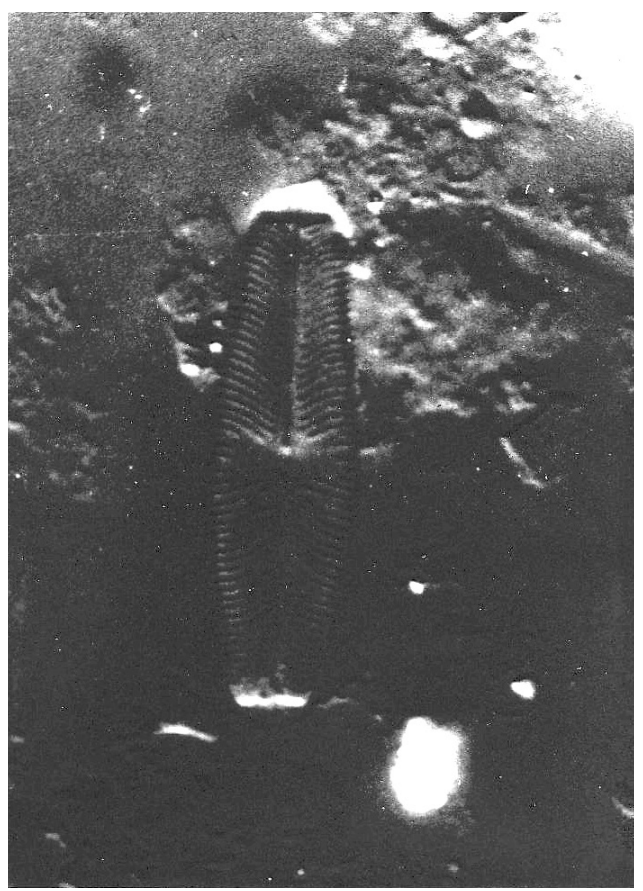

Navicula pupula fo. capitata

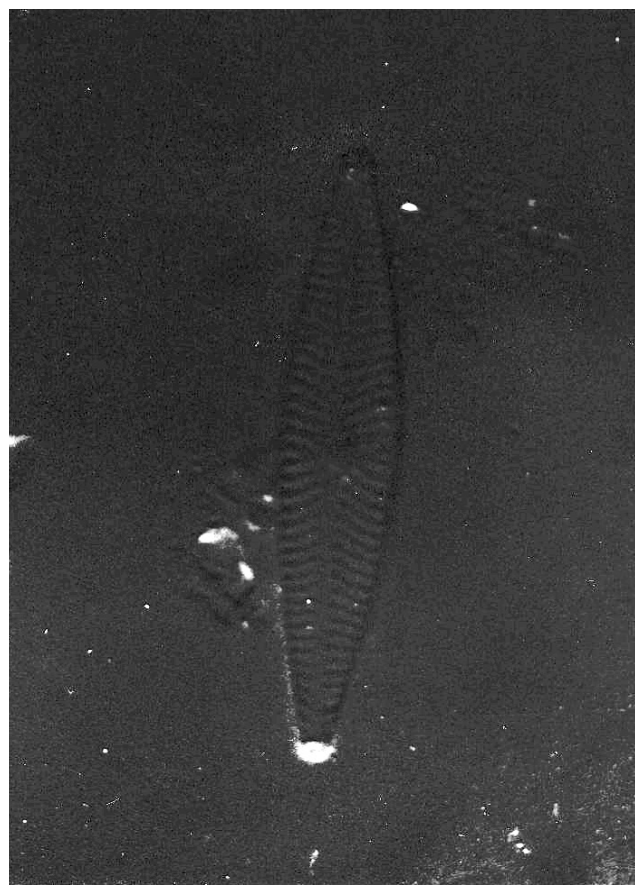

Navicula veneta

Figure 2. (Cont.) Periphytic diatoms recorded in the Linggi (s.s.) and Kundor rivers. 


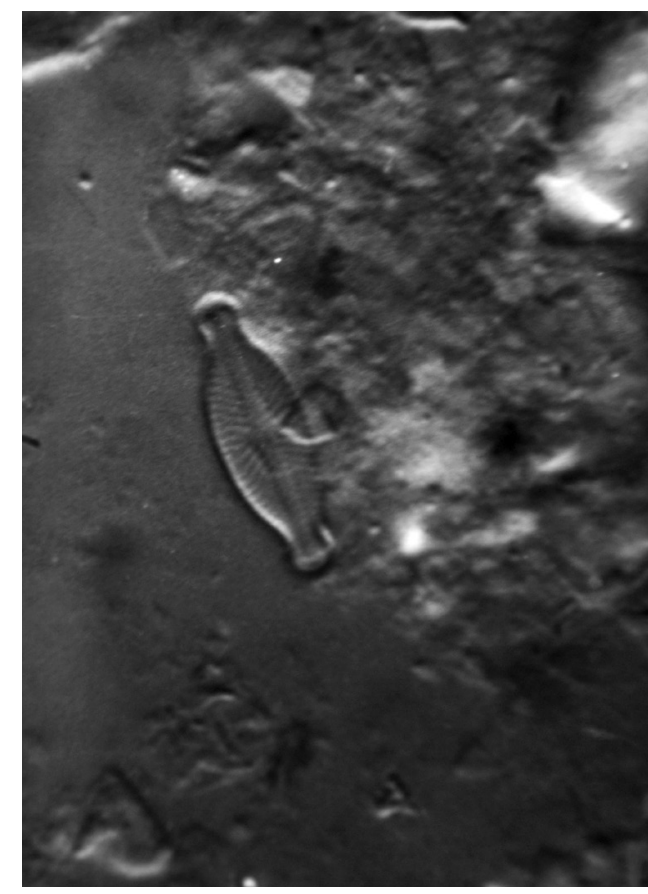

Navicula globosa

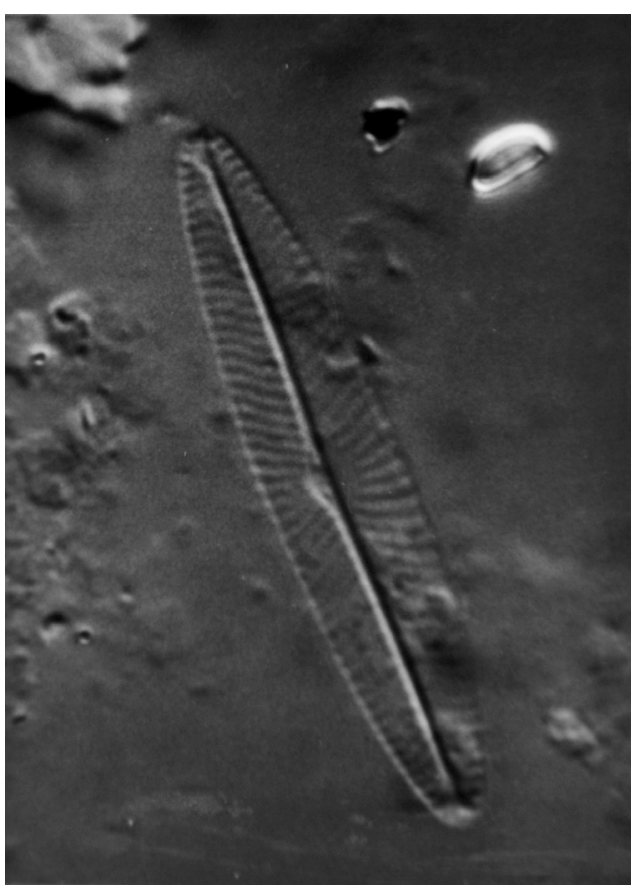

Navicula liboensis

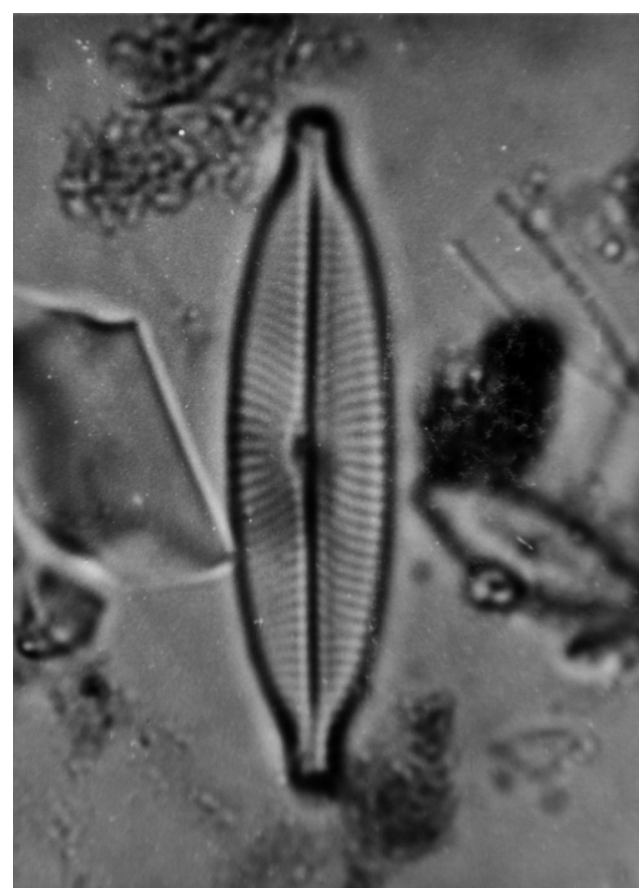

Navicula rhyncocephala

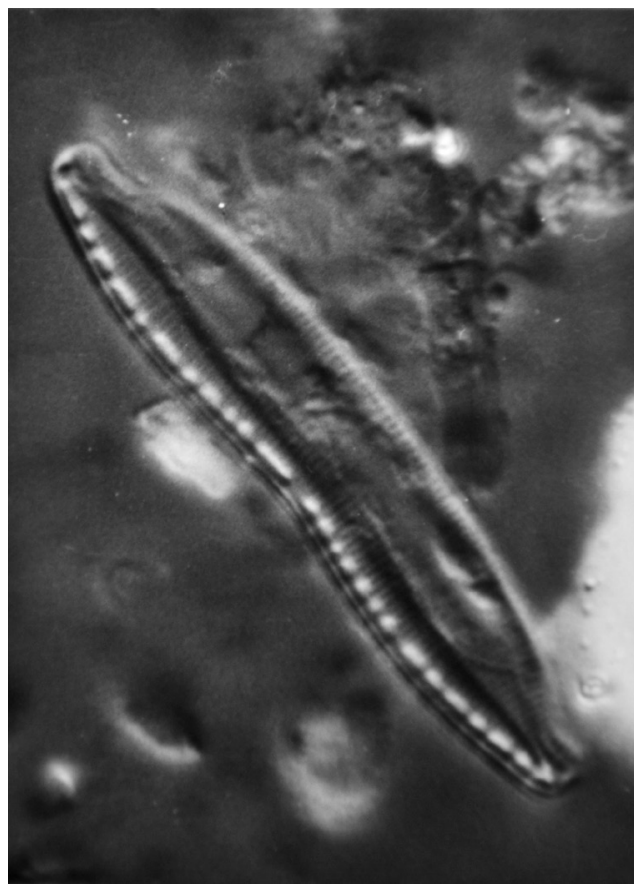

Nitzschia amphioxys

Figure 2. (Cont.) Periphytic diatoms recorded in the Linggi (s.s.) and Kundor rivers. 


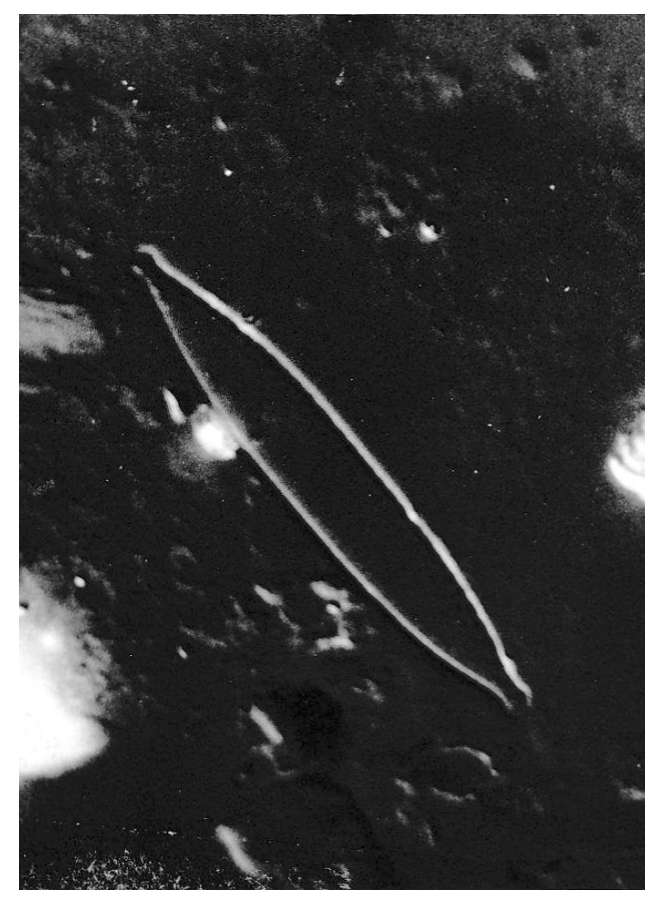

Nitzschia palea var. braunii

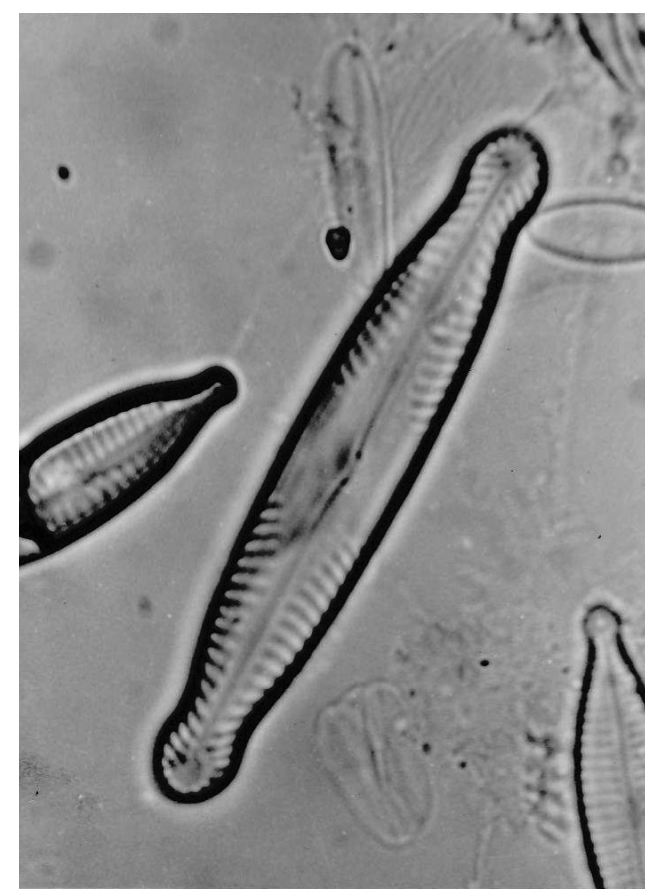

Pinnularia braunii var. amphicephala

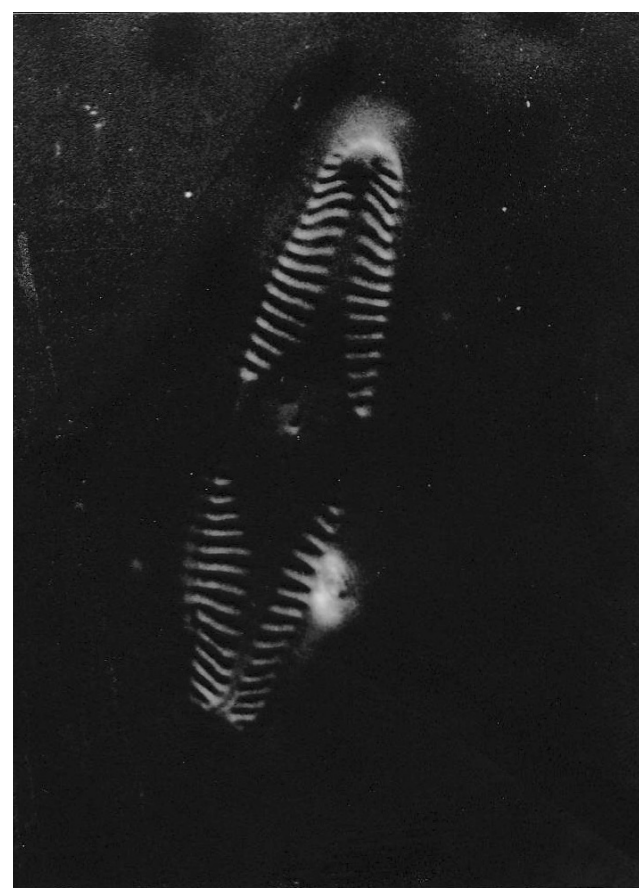

Pinnularia braunii

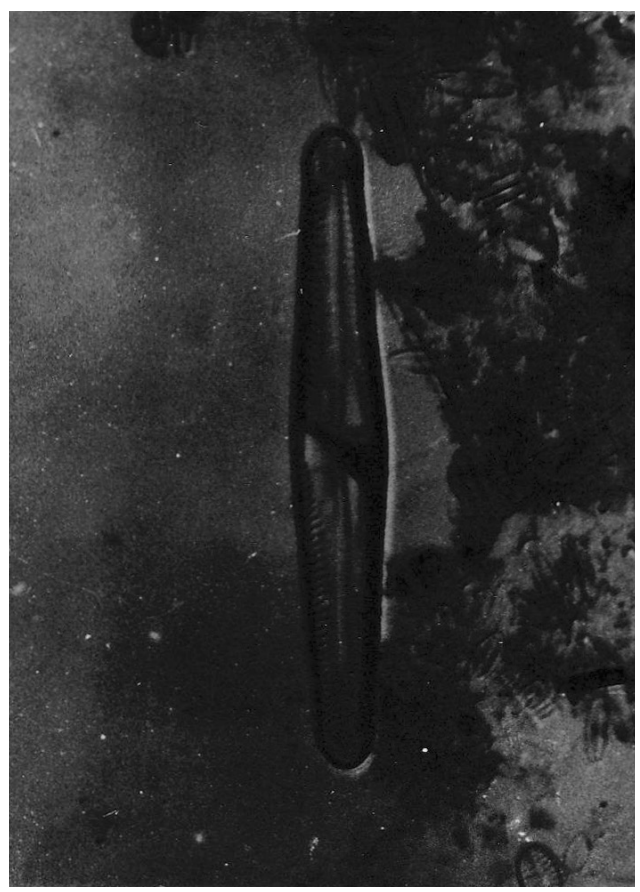

Pinnularia gibba var. interrupta

Figure 2. (Cont.) Periphytic diatoms recorded in the Linggi (s.s.) and Kundor rivers. 


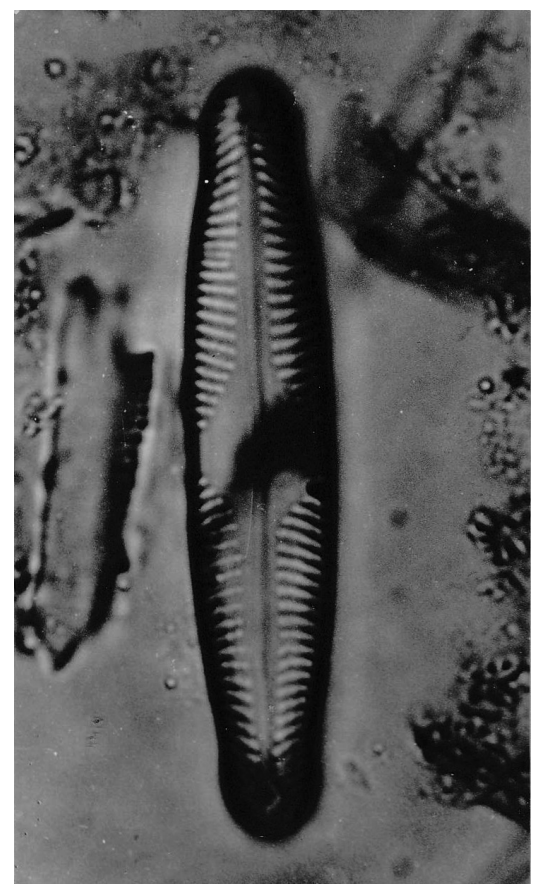

Pinnularia microstauron

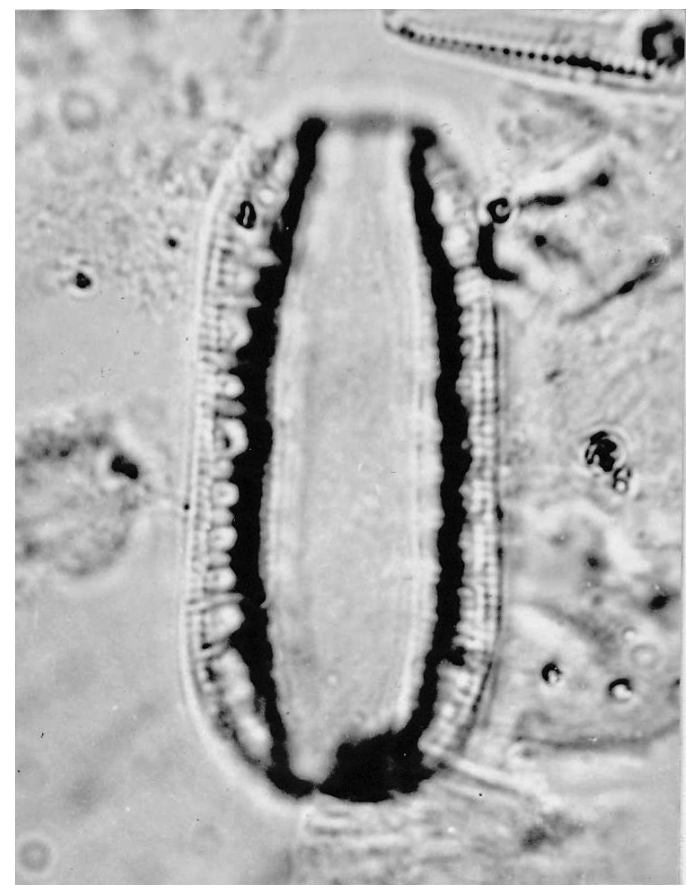

Surirella angusta

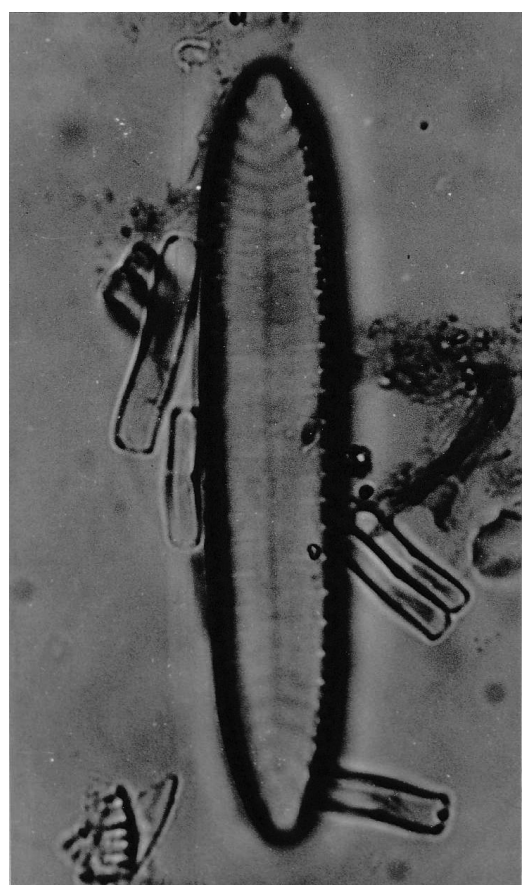

Surirella tenuissima

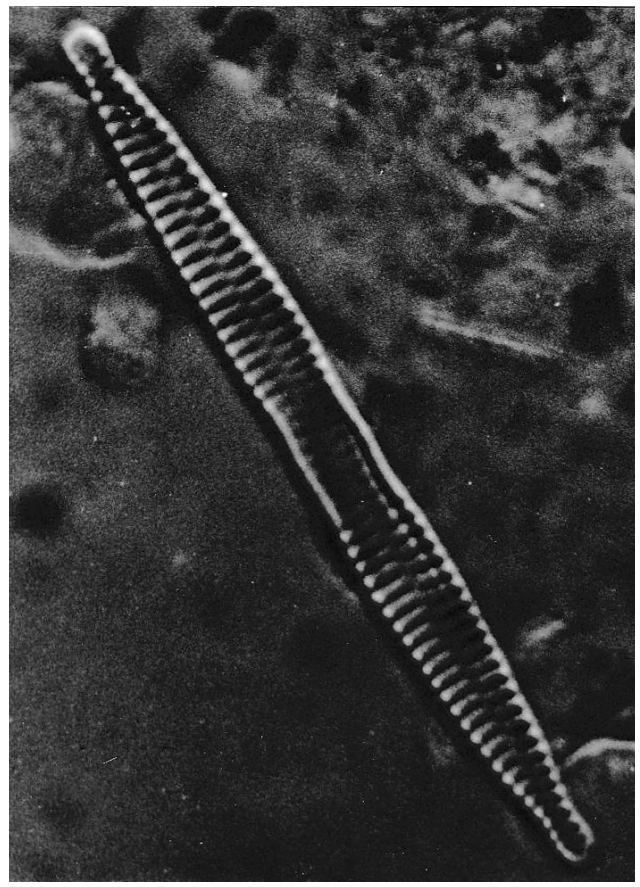

Synedra rumpens var. fragilarioides

Figure 2. (Cont.) Periphytic diatoms recorded in the Linggi (s.s.) and Kundor rivers. 


\section{CONCLUSION}

The periphytic diatoms were collected from both natural and artificial substrates comprised of 86 taxa ( 82 pennate and 4 centric forms) belonging to 21 genera. The number of diatom species observed between stations varied from 22 to 47 species under 8 to 16 genera respectively. On the whole, annual mean number of species varied from 8 to 22; the minimum species at Station 9, heavily polluted with rubber effluent and the maximum species at Station 3 mildly polluted with rubber effluent. The most common diatoms, if both the rivers combined were E. vanheurckii, G. parvulum, N. palea, $P$. braunii, N. cryptocephala, $A$. saxonica, $A$. minutissima and $P$. microstauron. The most abundant species were $E$. vanheurckii, N. palea, A. saxonica, G. parvulum and A. minutissim.

\section{Date of submission: June 2015 \\ Date of acceptance: August 2015}

\section{REFERENCES}

APHA, 1975, Standard methods for the examination of water and wastewater. 14th edn, American Public Health Association (APHA), Washington, D.C.

Arumugan, PT 1972, Limnological studies of a reservoir, Subang Lake, Klang, with special reference to algal blooms and pollution, Unpubl. B.Sc. (Hons.) Thesis, University of Malaya, Kuala Lumpur.

Bishop, JE 1973, 'Limnology of a small Malayan river - Sungai Gombak', W. Junk, The Hague.

Brown, SD \& Austin, AP 1973, 'Diatom succession and interaction in littoral periphyton and plankton', Hydro, vol. 43, nos. 3-4, pp. 333-356.

Castenholz, RW 1960, 'Seasonal changes in the attached algae of freshwater and saline lakes in the lower Grand Coulee, Washington', Limnol. Oceanogr, vol. 5, pp. 1-28.

Furtado, JI \& Mori, S (eds) 1982, 'The ecology of a tropical freshwater swamp, the Tasek Bera, Malaysia', Monographiae biologicae; vol. 47, W. Junk Publishers, The Hague, The Netherlands.
Hirano, M 1967, 'Freshwater algae collected by the joint Thai-Japanese biological expedition to Southeast Asia, 1961-1962', Nature and Life in S.E Asia, vol. 5, pp. 1-71.

Ho, SC 1973, 'The ecology of a lowland stream: Sungei Renggam with special reference to pollution', M. Sc. thesis, University of Malaya, Kuala Lumpur, Malaysia.

Hohn, MH \& Hellerman, J 1963, 'The taxonomy and structure of diatom populations from three eastern North American rivers using three sampling methods', Trans. Amer. Microcrop. Soc., vol. 82, pp. 250-329.

King, DL \& Ball, RC 1967, 'Comparative energetics of a polluted streams', Limnol. Oceanogr., vol. 12, pp. 27-33.

Mackereth, FJH, Heron, J \& Talling, JF 1978, 'Water analysis', Freshwater Biological Association Scientific Publication, no. 36.

Mizuno, T 1964, 'Illustrations of the freshwater plankton of Japan', Hoikusha Publ., Osaka, Japan.

Mizuno, T \& Mori, S. 1970, 'Preliminary hydrobiological survey of some Southest Asian inland waters', Biol. J. Linn. Soc., vol. 2, pp. 77-117.

Nather Khan, ISA 1985, 'Studies on the water quality and periphyton community in the Linggi River Basin Malaysia', Ph. D thesis, Uiversity of Malaya, Kuala Lumpur, Malaysia.

Nather Khan, ISA 1990a, 'Diatom distribution and inter-site relationship in the Linggi River Basin, Peninsular Malaysia, Malayan Nature Journal, vol. 44, pp. $85-95$.

Nather Khan, ISA 1990b, 'Assessment of water pollution using diatom community structure and species distribution - a case study in a tropical river basin', Int. Revue ges. Huydrobio., vol. 75, no. 3, pp. 317-338.

Nather Khan, ISA 1990c, 'The mineralogy and trace element constituents of suspended stream sediments of the Linggi River Basin, Malaysia', J. Southeast Asian Earth Sciences, vol. 4, no. 2, pp. $133-139$.

Nather Khan, ISA 1991a, 'Evaluation of effect of urban and industrial wastes on species diversity of diatom community in a tropical river basin, Malaysia', Hydrobiologia, vol. 224, pp. 175-184. 
Nather Khan, ISA 1991b, 'Sources of pollution and control strategies for the Linggi River Basin (Tropical), Malaysia, Chap XXXIX Environmental studies, in Proceedings of 9th Miami International Congress on Energy and Environment, vol. G, Nova Science Publishers Inc, New York, USA.

Nather Khan, ISA 1992a, 'A study on the impact of urban and industrial development on quality of rivers in the Linggi River Basin 1, morphometry and physical environment', Int. Revue ges. Hydrobiol., vol. 77, no. 2, pp. 203-223.

Nather Khan, ISA 1992b, 'A study on the impact of urban and industrial development on quality of rivers in the Linggi River Basin 2, chemical environment', Int. Revue ges. Hydrobiol., vol. 77, no. 2, pp. 203-223.

Nather Khan, ISA \& Haji Mohamed, M 1985, 'Freshwater Malaysia algae 2, the Diatoms', Nature Malaysiana, vol. 10, pp. 28-31

Nather Khan, ISA., Lim, RP \& Ratnasabapathy, M 1986a, 'Changes in river diatom community structure due to natural rubber effluent', Malaysian J. Sci., vol. 8, pp. 85-89.

Nather Khan, ISA, Lim, RP \& Furtado, JI 1986b, 'The impact of natural rubber effluent (SMR) on the distribution and species diversity of the diatom community in the Batang Penar River, Malaysia', in Proc. Int. Conf. Chem. Envir., eds JN Lester et al., Lisbon, Portugal 187-194.

Nather Khan, ISA, Furtado, JI \& Lim, RP 1987, 'Periphyton on artificial and natural substrates in a tropical river', Arch. Hydrobiol.Beih., Ergebn. Limnol.', vol. 28, pp. 473-484.

Nather Khan, ISA \& Lim, RP 1991, 'Distribution of metals in the Linggi River Basin with reference to pollution', Aust. J. Mar. Freshwater Res., 1991, vol. 42, pp. 435-439.

Nather Khan, I \& Firuza, BM 2010, 'Spatial and temporal variation of Silica in a disturbed tropical river basin', Sains Malaysiana, vol. 39, no. 2, pp. 189-198.

Nather Khan, I \& Firuza, BM 2012, Biological assessment of water pollution using periphyton productivity and standing crop in the Linggi River, Malaysia', International Review Hydrobiologia, vol. 97, no. 2, pp. 124-156.

Palmer, CM 1969, 'A composite rating of algae toleration organic pollution', Journal of Phycology, vol. 5, pp. 78.

Patrick, R \& Reimer, CW 1966, 'The diatoms of the United States. Exclusive of Alaska and Hawaii', vol. I, Mongor. Acad Nat. Sci. Phila., no. 13.

Prowse, GS 1962a, 'Diatom of Malayan freshwaters', Gard. Bull. Sing., vol. 19, pp. 1-104.

Prowse, GA \& Ratnasabapathy, M 1970, ‘A species list of freshwater algae from Taiping Lakes, Perak, Malaysia', Gard. Bull. Sing.', vol. 25, pp. 179-187.

Sladecek, V \& Sladeckova, A 1964, 'Determination of the periphyton production by means of the glass slide method', Hydrobiologia, vol. 23, pp. 125-158.

Sladecek, V 1963, ‘A guide to limnosaprobial organisms scientific papers from institute of chemical technology, Prague', Technology of water, vol. 7, no. 2, pp. 543-612.

Whitford, LA \& Schumacher, GJ 1963, 'Communities of algae in North Carolina streams and their seasonalrelations', Hydrobiologia, vol. 22, pp. 133-196. 